\title{
Cortical activation during executed, imagined, observed, and passive wrist movements in healthy volunteers and stroke patients
}

\author{
André J. Szameitat $^{(1,2)^{*}}$, Shan Shen ${ }^{(2,3)}$, Adriana Conforto ${ }^{(4)}$, Annette Sterr ${ }^{(2)}$ \\ (1) Department of Psychology, Ludwig Maximilians University, Munich, Germany \\ ${ }^{(2)}$ Department of Psychology, University of Surrey, Guildford, UK \\ ${ }^{(3)}$ School of Psychology and Clinical Language Sciences, University of Reading, Reading, UK \\ ${ }^{(4)}$ Hospital das Clínicas da Universidade de São Paulo and Albert Einstein Hospital, Sao Paulo, \\ Brazil
}

* Corresponding Author

Dr André J. Szameitat

Department of Psychology

Ludwig Maximilians University

Leopoldstrasse 13

80802 Munich

Germany email: szameitat [at] psy.lmu.de

phone: 0049-(0)89-2180 72521

fax: 0049-(0)89-2180 72538

\begin{abstract}
Motor imagery, passive movement, and movement observation have been suggested to activate the sensorimotor system without overt movement. The present study investigated these three covert movement modes together with overt movement in a within-subject design to allow for a fine-grained comparison of their abilities in activating the sensorimotor system, i.e. premotor, primary motor, and somatosensory cortices. For this, 21 healthy volunteers underwent functional magnetic resonance imaging (fMRI). In addition we explored the abilities of the different covert movement modes in activating the sensorimotor system in a pilot study of 5 stroke patients suffering from chronic severe hemiparesis. Results demonstrated that while all covert movement modes activated sensorimotor areas, there were profound differences between modes and between healthy volunteers and patients. In healthy volunteers, the pattern of neural activation in overt execution was best resembled by passive movement, followed by motor imagery, and lastly by movement observation. In patients, attempted overt execution was best resembled by motor imagery, followed by passive movement, and lastly by movement observation. Our results indicate that for severely hemiparetic stroke patients motor imagery may be the preferred way to activate the sensorimotor system without overt behavior. In addition, the clear differences between the covert movement modes point to the need for within-subject comparisons.
\end{abstract}

\section{Keywords}

Neurorehabilitation, movement simulation, motor imagery, movement observation, passive movement, overt execution

\section{Full reference (as of June 2012)}

Szameitat, AJ, Shen, S, Conforto, A, \& Sterr, A (2012). Cortical activation during executed, imagined, observed, and passive wrist movements in healthy volunteers and stroke patients. Neuroimage, 62 , pp. 266-280. DOI 10.1016/j.neuroimage.2012.05.009

For a reprint of the final pdf as published in the journal, please do not hesitate to contact szameitat@psy.Imu.de

Figures and tables can be found at the end of the manuscript. 


\section{Introduction}

Motor deficits remain one of the major, and often most debilitating deficits following brain damage. These motor deficits are typically treated with physical therapy. The latter, however, requires some residual abilities in the affected limb, which disregards these practices for patients suffering from complete or nearly complete loss of motor function. Thus, alternative approaches are needed to support recovery of the motor system without the need for overt behavior. Given the presumption that recovery is supported by an activation of the motor system (Kaneko, Murakami et al. 2003; Johnson-Frey 2004), the question arises how such an activation can be generated without actual overt movements. For this, at least three approaches have been suggested.

Before describing these approaches, we will define the relevant neuroanatomical structures in more detail. Since it is not finally decided which exact brain areas are relevant for motor rehabilitation, we decided to focus our investigations on the sensorimotor system, which may be subdivided into several areas: (a) lateral and medial premotor cortices (Brodmann's area (BA) 6 and 44) which are involved in the programming and planning of actions; (b) primary motor areas (BA 4) which are involved in the actual control of muscles; and (c) somatosensory cortices in the postcentral gyrus and inferior parietal lobe (BAs 1, 2, 3, 40) which are involved in the processing of the tactile and proprioceptive feedback of the action. In this manuscript we use the term "motor system" to refer to areas defined by (a) and (b), and the term "sensorimotor system" to refer to areas defined by (a), (b), and (c).

One approach to activate the sensorimotor system without overt movement is motor imagery, i.e. the mental simulation of a motor act. It has been suggested that kinesthetic motor imagery involves the same neural network as motor planning (Jeannerod 1994; Jeannerod and Frak 1999), which in turn is thought to rely on the same motor structures as motor execution (Johnson-Frey 2004; Sharma, Pomeroy et al. 2006; Munzert, Lorey et al. 2009). In support of this view, motor imagery shares a number of similarities with overt movement execution, such as behavioral (Decety and Jeannerod 1995) and physiological parameters (Kranczioch, Athanassiou et al. 2008; Kranczioch, Mathews et al. 2009), and, importantly, the functional neuroanatomical correlates (Decety 1996; Porro, Francescato et al. 1996; Lotze and Halsband 2006; Szameitat, Shen et al. 2007; Szameitat, Shen et al. 2007). In line with these findings, mental practice, i.e. training using motor imagery, has been successfully applied in sports training and rehabilitation (Feltz and Landers 1983; Jackson, Lafleur et al. 2001; Page, Levine et al. 2001; Lafleur, Jackson et al. 2002; Jackson, Lafleur et al. 2003; Johnson-Frey 2004; Braun, Beurskens et al. 2006; Cramer, Orr et al. 2007; Müller, Butefisch et al. 2007; Page, Szaflarski et al. 2009; Garrison, Winstein et al. 2010). Of the three suggested approaches, motor imagery is probably the most widely used approach in motor rehabilitation, most likely due to its early use in sports training.

A second approach is passive movement, i.e. the affected limb is passively moved by the therapist. Passive movement is thought to activate the sensorimotor system through afferences conveying proprioceptive information not only to sensory but also to motor cortices (Lemon and Porter 1976; Lemon 1999; Naito, Roland et al. 2002; DechaumontPalacin, Marque et al. 2008; Terumitsu, Ikeda et al. 2009). Previous studies showed in accordance with these ideas that the brain networks subserving passive movement and overt execution overlap strongly (Puce, Constable et al. 1995; Yetkin, Mueller et al. 1995; Weiller, Juptner et al. 1996; Carel, Loubinoux et al. 2000) (but see Mima, Sadato et al. 1999). Again, evidence suggests that passive movement can be successfully applied in motor rehabilitation (Hesse, Bertelt et al. 1995; Lewis and Byblow 2004; Lindberg, Schmitz et al. 2004; Ward, Brown et al. 2006; Dechaumont-Palacin, Marque et al. 2008) (but see Lotze, Braun et al. 2003). Because the overt movement of the limb in passive movement is not controlled by the participant, we consider the passive movement condition used in the present manuscript as a covert movement mode as well.

A third approach is movement observation, i.e. patients watch another person or a video clip of a moving limb. It has been argued that movement observation activates the human mirror neuron system, which consists of neurons responding to both, the overt execution of an action and the observation of that same action performed by somebody else (Pomeroy, Clark et al. 2005; Ertelt, Small et al. 2007; Iacoboni and Mazziotta 2007; Gazzola and Keysers 2009; Rizzolatti and Sinigaglia 2010; Roosink and Zijdewind 2010). Initial evidence suggests that movement observation can also be successfully applied in rehabilitation (Ertelt, Small et al. 2007; Celnik, Webster et al. 2008; Stefan, Classen et al. 2008).

Given that all three covert movement modes, passive movement, imagery and observation, appear to activate the sensorimotor system in a way that makes the approaches suitable for neurological intervention, the question arises how these approaches directly compare to each other. Such knowledge is important in the endeavor to develop and refine covert movement based interventions that are optimized for patient parameters such as lesion or motor deficit characteristics.

The general idea of covert movement modes is supported by a substantial body of evidence. However, the majority of this literature investigated only one mode of covert movement mode or, at most, compared two modes, usually motor imagery and observation (Clark, Tremblay et al. 2003; Filimon, Nelson et al. 2007; Iseki, Hanakawa et al. 2008; Lui, Buccino et al. 
2008; Munzert, Zentgraf et al. 2008; Wang, Wai et al. 2008; Piefke, Kramer et al. 2009; Roosink and Zijdewind 2010; Macuga and Frey 2012). Therefore inferences between studies have to be made when comparison between all modes of covert movement are to be conducted. The latter is not ideal because studies differ in their samples, their tasks, and other methodological aspects. The present study therefore aimed to fill this gap by concurrently investigating all four movement modes in a repeated-measure design to establish the similarities and discrepancies of their neuroanatomical signatures. Here we report data from 21 healthy volunteers (main study) and 5 patients with severe left-sided hemiparesis after stroke (pilot study). Because the suggested rehabilitation approaches are most relevant for severely hemiparetic patients with no or low residual movement abilities, we used a simple flexion-extension movement of the wrist as the basis for all conditions.

\section{Methods}

\subsection{Participants}

The main experiment was conducted with 21 neurologically healthy right-handed volunteers (11 male; mean Oldfield score $=84$, range 64-100, Oldfield 1971) aged 19-43 (mean 25). This group is referred to as healthy volunteers (HVs). For the case study, 5 patients with chronic severe left hand hemiparesis following right hemispheric lesions caused by stroke were tested. To avoid confusion, we will refer to the healthy volunteers with the abbreviation HVs, to the patients as patients, and to both groups as participants. Inclusion criteria of patients comprised chronicity $>1$ year and low residual movement abilities in the affected wrist. Exclusion criteria were contraindication to MRI, cognitive impairments, and neurological disorders. For further details see Tab 1 and Fig 3. The study was approved by the University of Surrey ethical review board. All participants gave written informed consent prior to scanning.

\subsection{Task and procedure}

Participants were lying in the functional magnetic resonance imaging (fMRI) scanner in supine position. Task instructions and stimuli were presented on a projection screen via a mirror system.

All conditions involved overt or covert motion of the hand and comprised an alternating flexionextension movement of the wrist. The person's hand was secured (using Velcro straps) on a moveable flap (Fig 1) to control the trajectory and to limit the extent of the movement to $40^{\circ}$ upward flexion. At rest, the participant's hand was in line with the forearm and the flap rested on two non-magnetic force sensors.

There were 8 conditions presented blockwise: (1) For resting baseline with closed eyes (Base $(\mathrm{C})$ ) participants were instructed to stay at rest and to keep their eyes closed; (2) For resting baseline with open eyes $(\mathrm{Base}(\mathrm{O}))$ participants were instructed stay at rest and to fixate a cross in the center of the screen; (3) For overt execution (Execution) HVs were instructed to flex and extend their wrist with a frequency of $1 \mathrm{~Hz}$ paced by an auditory stimulus presented via headphones. The auditory stimulus was presented at 2 $\mathrm{Hz}$ and indicated when the hand had to be at the bottom and top position of each movement cycle. Patients were instructed to give their best to execute the movement. However, they were discouraged to try so hard that movement of other body parts would occur; (4 \& 5) For motor imagery with the right/left hand (Imagery $(\mathrm{R}) / \operatorname{Imagery}(\mathrm{L}), \quad$ respectively) participants were instructed to engage in kinesthetic motor imagery (Stinear, Byblow et al. 2006; Guillot, Collet et al. 2009) of the same wrist movement as in Execution; (6) For movement observation (Observation) participants were instructed to attend to a video clip showing a hand (Fig 2) which moved in the same way as required by the Execution condition; ( $7 \& 8)$ There were two passive movement conditions. For both conditions, participants were instructed to relax their hand and let it be moved freely. In particular, they were instructed not to help, aid, or support the movement. In one condition (PassiveMove(Flap)) the participant's hand rested on the flap, which was moved up and down by the experimenter; In the second condition (PassiveMove(Exp)) the participant's hand rested on the experimenter's hand, which was moved up and down by the experimenter. Since subsequent analyses of the two passive movement conditions revealed no differences they were collated (PassiveMove). Onset of all conditions was predictable to the subjects, since conditions always started directly after an instruction period lasting $10 \mathrm{~s}$.

To aid the experimental procedure additional stimuli were presented. To cue the experimenter the pacing of movement during PassiveMove, the screen background switched colors between yellow and light green at $2 \mathrm{~Hz}$ and in synchrony with the auditory pacing sound heard by the participants (cf. Fig. 2). Participants were able to see this as a light change in luminance through the closed eye lids. This change in background color was incorporated in all conditions to ensure similarity of physical stimulus characteristics. Furthermore, participants had their eyes closed during all conditions except for Observation, $\operatorname{Base}(\mathrm{O})$, and the instruction periods between two blocks. An auditory cue was used to signify participants to open their eyes. In addition, each instruction screen was presented on a bright white screen, which was easily recognized through closed eye lids.

Blocks lasted 20 seconds and contained 20 overt or covert flexion/extension movements respectively. Blocks were separated by a 10 second instruction period. Conditions were presented in a pseudorandomized order. For HVs, each of the eight conditions was repeated eight times and the experiment was split into two functional runs (four repetitions per condition), each lasting $16 \mathrm{~min}$. Between the two runs a high-resolution anatomy scan was performed, resulting in a break of 5-6 min for the HVs. 
For the patients the procedure was identical except for the following. First, because for all patients the attempt of overt execution required tremendous effort, movement artifacts were likely to occur. In order to avoid that excessive overt movement reduced data quality of the covert movement conditions, Execution was performed in a separate functional run after all other conditions had been acquired. Second, to shorten the experiment each condition was presented only six times. Thus, there were two functional runs lasting 10.5 min each during which all above described conditions except Execution were presented. In addition, a third functional run lasting $6.5 \mathrm{~min}$ was administered, in which seven $\operatorname{Base}(\mathrm{C})$ blocks were presented alternating with six Execution blocks. The high-resolution anatomy was acquired between the first two runs.

To test for overt movements of the non-acting hand, participants held a force sensitive grip in it (Hou, Shen et al. 2005). The acting hand (right hand in HVs, left hand in patients) lay, as described above, on a flap which rested on similar force sensors as used in the grip of the non-acting hand. Force data were sampled continuously throughout all conditions with a frequency of approximately $250 \mathrm{~Hz}$. The sensors are highly sensitive to force changes and are able to detect force variations not noticeable by visual inspection. In addition, the experimenters marked blocks with visible movement (e.g. of other body parts) and if participants were not fully passive during PassiveMove. Such blocks were excluded from statistical analyses.

\subsection{Questionnaires}

HVs and patients filled out a self-developed questionnaire immediately after the fMRI scan. The questionnaire contained questions about the different conditions, such as quality of imagination or selfperceived overt movement during the non-movement conditions. For details, see Results section.

Patients were administered a number of further tests assessing motor and general impairments. These were the Mini-Mental State Examination Test (MMSE), the Frenchay arm test, the Stroke Impact Scale (SIS, version 3.0), and three (partly) selfdesigned tests. Two of these three tests assessed patients' abilities to imagine motor acts and were based on the methods described in Sirigu et al. (1996). The first test mainly assessed visual imagery abilities and asked patients to imagine their own hand in a specific position (e.g. fingers pointing up, back of hand facing you) and to indicate the location of a particular finger (e.g. is your thumb on the left or right side?). The test was conducted for both hands separately, beginning with the affected hand.

The second imagery test assessed the abilities of kinesthetic motor imagery and asked patients to repeat six movements which differed in their motor complexity. In separate runs, patients imagined each movement with their right and left hand and overtly executed them with their unaffected hand. As the movements were quite short, for a single run participants were asked to repeat each movement five times in immediate succession. The time taken for each run was taken, with the assumption that the more complex movements take longer to imagine and execute (Decety and Jeannerod 1995; Sirigu, Duhamel et al. 1996). Patients performed three runs of each condition (left/right imagery, execution), and the average of the three runs was used for analysis. However, although we observed differences in the time taken to execute and imagine different movements, these timing differences not always reflected the postulated complexity. To account for this, we analyzed the data in the following way: Taking Execution (using the unaffected hand) as the gold standard, we correlated right and left hand imagery separately with Execution. If a patient is generally unable to imagine kinesthetically, both correlations should be low. If imagery is affected by the lesion, only imagery of the affected hand should be uncorrelated with Execution. If imagery was unaffected, both correlations should be high.

Finally, we assessed the movement abilities and potential sensory impairments. To test for movement abilities, patients sat comfortably on a chair and were asked to produce flexion-extension movements with each finger, the wrist, the elbow, and the shoulder. The degrees of movement around the joint were noted. To test for sensory impairments, patients were asked to close their eyes and the experimenter touched different points on the finger, arm, and shoulder. In addition, the experimenter moved the finger and wrist. Patients indicated whether they felt touch / movement or not.

\subsection{MRI procedure}

Imaging was carried out at the Royal Holloway University London, UK, using a 3T scanner (Trio, Siemens, Erlangen, Germany) equipped with an eight channel array head coil. Participants were supine on the scanner bed, and cushions were used to reduce head motion. 36 axial slices $(192 \times 192 \mathrm{~mm}$ field of view (FOV), 64x64 matrix, 3x3 $\mathrm{mm}$ in-plane resolution, $4 \mathrm{~mm}$ thickness, no gap, interleaved slice acquisition) were acquired using a BOLD sensitive gradient echo EPI sequence (TR $2 \mathrm{~s}$, TE $30 \mathrm{~ms}, 90^{\circ}$ flip angle). High-resolution whole brain images were acquired from each participant using a T1-weighted MPRAGE sequence (TR $1830 \mathrm{~ms}$, TE $4.43 \mathrm{~ms}, 11^{\circ}$ flip angle, 176 slices, $256 \times 256 \mathrm{~mm}$ FOV, 1x1x1mm voxel size). For HVs, two functional runs with 480 volumes each were acquired, with each volume sampling all 36 slices. For patients, three functional runs were acquired, the first two runs consisting of 315 scans, the third (assessing Execution) of 195 scans.

\subsection{Data analysis}

The data were analyzed using SPM5 (http://www.fil.ion.ucl.ac.uk/spm/software/spm5/).

First, the origin of the anatomical and functional images was manually set to the anterior commissure and all images were reoriented. To correct for movements, all functional volumes were spatially realigned to the first functional volume, and signal 
changes due to head motion and magnetic field inhomogeneities were corrected (Andersson, Hutton et al. 2001). Anatomical and functional images were normalized to MNI space, and functional data were spatially smoothed using a Gaussian kernel with a FWHM of $8 \mathrm{~mm}$. Normalization and registration success was validated by visual inspection. This confirmed that the images of all participants, including patients, were normalized and registered correctly.

To avoid confounds, experimental blocks were excluded from the analysis as follows. For HVs, blocks in which HVs moved or were not fully passive during PassiveMove were excluded. Patients showed no movement during conditions other than Execution and did not actively support the movement during PassiveMove. However, Execution blocks were excluded if movement of other body parts than the wrist occurred.

Statistical analysis was based on a voxel-wise least squares estimation using the general linear model for serially autocorrelated observations (Friston, Holmes et al. 1995). A temporal high-pass filter with a cutoff frequency of $1 / 300 \mathrm{~Hz}$ was applied. For HVs, individual contrast maps were calculated for all contrasts of interest (see Results section) and the second-level analysis was based on random-effects ttests. Due to the small sample size, for the patients we employed a fixed-effect model in which all data of all participants are entered into the analysis and calculation of contrasts. For the patients, baseline comparisons were based on the baseline the condition originated from, i.e. Execution - Base $(C)$ of run 3, and all other conditions $-\operatorname{Base}(C)$ of runs 1 and 2 . In addition, to control for the effect of run in across-run comparisons we first related each condition to its runspecific baseline. This resulted in interaction contrasts (denoted (I)) such as [(Imagery - Base(C) of runs 1 and 2$)-($ Execution $-\operatorname{Base}(\mathrm{C})$ of run 3$)]$. All resulting statistical parametric t-maps were thresholded at $\mathrm{p}<$ .05 (FWE corrected for multiple comparisons at voxel level), resulting in thresholds for HVs of $\mathrm{t}(20)=6.6, \mathrm{p}$ $=0.000002$ (uncorr) and for patients of $\mathrm{t}(3967)=4.71$, $\mathrm{p}=0.000003$ (uncorr).

The similarity of the activation patterns of the different covert movement modalities with overt / attempted execution was evaluated by (1) visual inspection of the respective baseline contrasts (e.g. whether Execution-Baseline and Imagery-Baseline resulted in visually comparable activation patterns or not, cf. Fig 4), (2) direct condition contrasts presented in the Supplementary Materials (e.g., if Execution Imagery resulted in virtually no activation, while Execution - Observation resulted in profound sensorimotor activation, we concluded that Imagery and Execution were more similar than Observation and Execution and consequently that Imagery resembled Execution better than Observation; cf. Fig S1 and S2), and (3) by visual inspection and comparison of the activation peaks presented in Tab 3 and 4).

Fixed-effect models, as applied for the patient data, have more statistical power than random-effect models and are therefore an approach to avoid type-II (beta-) errors when sample size is small (Friston, Holmes et al. 1999). However, because individual patients may bias the group statistics, conclusions from fixed-effect models are limited to the investigated sample and cannot be generalized to the population. To provide the reader with more details about the consistency of the findings, tables of patient data include the number of patients showing a particular effect. More specifically, we determined for each patient the activation peak in a cube of $5 \times 5 \times 5$ voxel (i.e., the two neighboring voxel in each direction) surrounding the group peak and report whether the beta-value of this voxel is in the expected direction (i.e., positive beta-value in case of activation at group peak) and whether it is significantly different from zero $(\mathrm{p}<.05)$. As a more conservative measure, these two values (number of patients showing voxels with expected direction and statistically significant voxels) were determined in each patient also for the group peak voxel itself (values in brackets).

All stereotaxic coordinates are reported in MNI space. Anatomical locations and Brodmann's areas were preferentially determined using the SPM Anatomy toolbox version 1.6 (Eickhoff, Stephan et al. 2005). If no probability information was available for a given peak, we converted the MNI coordinates into the space of Talairach \& Tournoux (1988) using the script mni2tal (Brett, Anton et al. 2002) and used the Talairach Daemon (Lancaster, Rainey et al. 1997; Lancaster, Woldorff et al. 2000) to determine anatomical structure and Brodmann area. These peaks are noted in the tables with ${ }^{(1)}$. If neither SPM Anatomy toolbox nor Talairach Daemon clearly identified location and cytoarchitectonic area, the anatomical location was determined manually by visual inspection supported by several atlases. These peaks are noted in the tables with ${ }^{(2)}$. In the results section cytoarchitectonic areas are given in brackets.

Lesion volume in patients was determined using an automatic approach (Shen, Szameitat et al. 2008) before and after normalization, i.e. in native and MNI space. Lesion volume in native/MNI space for patient 1 was $42277 / 49890 \mathrm{~mm}^{3}$, for patient $235060 / 49147$ $\mathrm{mm}^{3}$, for patient $371018 / 85748 \mathrm{~mm}^{3}$, for patient 4 $1506 / 2136 \mathrm{~mm}^{3}$, and for patient $51589 / 2339 \mathrm{~mm}^{3}$ (Fig 3, Tab 1).

\section{Results}

\subsection{Force data}

To test whether participants moved their wrist during the different conditions, we compared each condition with its respective baseline (i.e., Observation vs. Base(O); all other conditions vs. Base(C)). In HVs, the most significant result was that less force was exerted by the right hand during Execution $(\mathrm{t}(20)=5.653 ; \mathrm{p}<.001)$. This was expected because participants actively lifted the flap from the force sensors. In addition, participants exerted slightly more force $(0.05 \mathrm{~N})$ with the right hand during Imagery $(\mathrm{R})(\mathrm{t}(20)=2.118 ; \mathrm{p}<.05)$ as compared to 
Base $(\mathrm{C})$. All other comparisons with the respective baseline conditions, including all comparisons of the left hand, were not significant (all $\mathrm{p}>.05$ ).

In patients, there were no effects for the left (affected) hand resting on the flap (all $\mathrm{t}(4)<1.819$, all $\mathrm{p}>.143$ ). With the right hand (holding the grip) patients exerted more force $(1.21 \mathrm{~N})$ during PassiveMove as compared to $\operatorname{Base}(\mathrm{C})(\mathrm{t}(4)=3.055, \mathrm{p}$ $<.05)$. As a second effect, patients exerted less force $(0.92 \mathrm{~N})$ during Observation as compared to $\mathrm{Base}(\mathrm{O})$ $(\mathrm{t}(4)=-3.044, \mathrm{p}<.05)$.

\subsection{Questionnaire data $(H V s)$}

To test whether participants' subjective perception of performance differed between conditions, they rated their performance in each condition after the experiment. One set of items assessed whether participants had the impression that the executed, observed, imagined, and passive movements were in synchrony with the pacing sound. Generally, HVs perceived a high level of synchrony (lowest item 5.7 on a 1[fully asynchronous] to 7[full synchrony] scale). Nevertheless, using non-parametric Wilcoxon Signed Rank tests some significant differences occurred. In particular, imagery (combined for right and left hand) (rating 5.8) was rated to be slightly less in synchrony than Execution (rating 6.3; $\mathrm{Z}=2.495, \mathrm{p}<.05$ ) and Observation (rating 6.6; $\mathrm{Z}=2.996, \mathrm{p}<.005$ ). In addition, Passive Movement (5.7) was rated to be less in synchrony than Observation $(6.6 ; \mathrm{Z}=2.564$; $\mathrm{p}<$ $.05)$. A further set of items assessed whether the perceived asynchrony manifested as being slower or faster than the pacing sound (scale 1[slower] to 4 [sometimes slower, sometimes faster] to 7 [faster]). For these items, however, no significant differences were found (all $\mathrm{Z}<1.000$, all $\mathrm{p}>.317$ ). Taken together, these results suggest that HVs perceived their performance in good synchrony with the pacing sound. In addition, deviations from synchrony were balanced between being too fast and too slow. This pattern makes it unlikely that functional neuroanatomical differences are caused by differences in movement frequency or speed.

Regarding quality of imagination (scale 1[very easy to feel] to 7[very hard to feel]), HVs answered that the kinesthetic motor imagery was rather easy to feel (rating 2.5 for right hand imagery; 3.6 for left hand; this difference was significant with $\mathrm{Z}=2.641, \mathrm{p}$ $<.01)$.

HVs were confident that active movement (scale 1 [no movement] to 7 [strong movement]) of their hands during Imagery (rating 1.8), Passive Movement (1.7), and Observation (1.9) was minimal (conditions did not differ from each other; all $\mathrm{Z}<.5$; all $\mathrm{p}>$.617).

Finally, HVs paid attention to the video clip during Observation (rating 2.1 on a scale 1[full attention] to 7 [no attention]), and generally maintained a high state of concentration during the experiment (rating 2.7 on a scale 1 [very high] to 7 [very low]).

\subsection{Questionnaire data (Patients)}

Patients filled out the identical questionnaire as the HVs. However, data of patient 3 were questionable as he, for instance, also filled out items which were not applicable to him. All tests were therefore calculated twice, once with all patients $(\mathrm{N}=5)$, and once without the mentioned patient $(\mathrm{N}=4)$. Probably due to the small sample and frequent missing values, no statistical test reached significance (for $\mathrm{N}=4$ and $\mathrm{N}=5$ ) and we report the data only descriptively (for $\mathrm{N}=4$ ).

Except for Execution (rating 2) patients felt that the movements were in good synchrony with the pacing sound (ratings Imagery 5.5, Passive Movement 6.75, Observation 6.5). Regarding the nature of asynchrony, Imagery (rating 3.5) and in particular Execution (rating 2.67) were perceived to be too slow (probably due to unfortunate placement of the items on the questionnaire, patients did not answer this question for Observation and PassiveMove). Thus, with the possible exception of Execution, it is unlikely that differences in the neuroanatomical correlates are caused by differences in the frequency or speed of the movements.

Regarding quality of imagination, patients reported that the kinesthetic motor imagery was rather easy to feel with the right (unaffected) hand (rating 3) and neutral to slightly hard to feel with the left (affected) hand (rating 4.5). Patients were confident, that active movement of their hands during Imagery (rating 2), PassiveMove (1.9), and Observation (1) was minimal. Finally, patients paid attention to the video clip during Observation (rating 1.5), and generally maintained a high state of concentration during the experiment (rating 2).

\subsection{Stroke Impact Scale}

Patients filled out the stroke impact scale (SIS, version 3.0) after the scanning session. Each domain of the SIS was transformed to resemble a percentage score between 0 and 100, with 100 being of positive valence (e.g., full strength in limb, activity not affected, activity not difficult). Results showed that use of the affected hand was virtually impossible (domain 7 ; mean $1 \%$, range $0-5 \%$; cf. Table 2 ). In most participants the stroke mainly affected only the arm and partly the ability to walk. Patients stated only small impairments regarding non-motor aspects. For full details see supplementary online material.

\subsection{Overt movement during Execution (Patients)}

Overt movement produced by the patients in the Execution condition during the fMRI scan was assessed by the experimenter. In detail, movement of finger, wrist, lower arm/elbow, upper arm/shoulder, and other body parts were rated on a scale from 0 [no movement] to 5 [strong movement; for the wrist the required movement]. Patients generally showed very little movement of the wrist (in $57 \%$ of the blocks with a mean intensity of 1.1 ; range $0.8-1.5$ ) or fingers (36\% of blocks; mean 1.1; range $0-1.5$ ), but the effort to produce the movement resulted in movement of other body parts such as the elbow (50\% of blocks; mean 1.6; range $0-2$ ) or shoulder (30\% of blocks; 
mean 1.1; range 0-1.5). Three patients showed movement in mouth, leg, foot, and/or right wrist with intensities not exceeding 1.5. For further details see supplementary materials.

\subsection{Imagery abilities}

Patients performed two tests to assess their abilities to imagine motor acts, see Methods for details. The first test (Tab 2) mainly assessed visual imagery abilities and indicated that three patients had no problems with visual imagery at all (scoring 100\% for both hands). Patient 3 made one mistake for each hand, which is not suggestive of a particular impairment of imagery of the affected hand. Patient 2 , however, made two mistakes with the affected hand, which may indicate a slight impairment in visual imagery abilities.

In the second test assessing abilities of kinesthetic motor imagery, patients imagined and executed finger movements of different complexity. If kinesthetic imagery is intact, execution times between imagery and execution should be correlated. Results demonstrated (Tab 2) that three patients showed high correlations between imagery of the affected hand and overt execution with the good hand, while two patients may have had problems with kinesthetic imagery with the affected hand (Patient 1) or both hands (Patient 5). Note that correlations rarely were significant, most probably due to the small sample.

\subsection{Movement abilities and sensory deficits}

All patients produced no or only minor voluntary movements of the fingers and wrist, while some patients were able to produce more movement in the elbow and shoulder (Tab 2). No patient had sensory impairments in the shoulder, upper arm, and elbow (Tab 2). Three patients showed moderate sensation impairments in the wrist, hand, and fingers, i.e. a higher pressure to feel touch and larger movement to feel movement were required.

\section{8 fMRI Results Healthy Volunteers (HVs)}

Below, results are presented in the following way. First, each condition is described with the comparisons condition - Base, condition - Execution, and Execution - condition. Second, each condition is compared to the remaining conditions, i.e. Imagery(R), PassiveMove, and/or Observation. Finally, results are briefly summarized. Cytoarchitectonic areas are given in brackets. Only sensorimotor activations are reported (see supplementary online materials for a full description). Figure 4 and Table 3 present data of the baseline comparisons, for a figure showing all pairwise comparisons see supplementary online materials. For imagery, we report only $\operatorname{Imagery}(\mathrm{R})$ but not Imagery(L), because HVs performed all other tasks with the right hand.

\subsubsection{Execution}

The comparison Execution - Base $(\mathrm{C})$ activated the left postcentral gyrus $(4 \mathrm{p}, 1)$, with the activation slightly extending into the left precentral gyrus (6). In addition, the left supplementary motor area (SMA; medial 6) was activated extensively, with the activation reaching inferiorly into the middle cingulate cortex. Furthermore, bilateral activation was evident in the rolandic operculi (OP1).

In the comparisons Execution - Imagery(R) and Execution - Observation, an activation pattern highly comparable to Execution - Base(C) was observed. Thus, Execution activated sensorimotor cortices much stronger than Imagery(R) and Observation. Opposed to this, the Execution - PassiveMove contrast revealed only small circumscribed activations in the left sensorimotor cortices, indicating a high level of similarity between Execution and PassiveMove. The latter three contrasts are described in more detail below.

Taken together, Execution activated an expected sensorimotor network of brain areas and this activation was stronger than in all other conditions except for PassiveMove.

\subsubsection{PassiveMove}

The comparison PassiveMove - Base $(\mathrm{C})$ resulted in a virtually identical activation pattern as compared to Execution - Base $(C)$. In particular, activation was also evident in the left postcentral gyrus (3b), but this activation extended slightly more anterior than in Execution - Base $(C)$ and included the precentral gyrus $(4 a, 6)$ as well. The contrast PassiveMove - Execution revealed only a difference in the anterior cingulate cortex (BA 24), but no difference in the left pre- or postcentral gyrus. The reversed contrast, i.e. Execution - PassiveMove showed that the activations in the left sensorimotor cortex, i.e. left pre- (6) and post-central (2) gyri, were slightly but significantly stronger in Execution as compared to PassiveMove.

PassiveMove - Imagery $(\mathrm{R})$ revealed activation in the left sensorimotor cortex, i.e. precentral gyrus (4a, 6 ), postcentral gyrus (2), and rolandic operculum (OP1). In addition, the inferior part of the SMA was activated. PassiveMove - Observation showed basically the same pattern of activation, except that the whole SMA was activated.

Taken together, PassiveMove activated the same areas as Execution, but slightly weaker. In addition, PassiveMove activated sensorimotor areas stronger than Imagery(R) and Observation.

\subsubsection{Imagery $(R)$}

The comparison Imagery $(\mathrm{R})-\operatorname{Base}(\mathrm{C})$ activated the SMA (6) comparably to Execution - Base(C). In addition, premotor areas in the precentral gyrus (6), superior frontal gyrus (6), and bilateral inferior frontal gyri (44) were activated. Although these activations were not evident in Execution - Base $(C)$ at the chosen threshold, they failed to reach significance in the direct comparison Imagery(R) - Execution. Instead, there were differences mainly in other prefrontal areas, such as the anterior inferior frontal gyrus, orbital gyri, and the anterior superior frontal gyrus. The contrast Execution - $\operatorname{Imagery}(\mathrm{R})$ revealed a 
network highly comparable to Execution - Base(C), i.e. left postcentral gyrus (4a, 1, 2, 3b), left SMA (6) extending inferiorly into the mid-cingular cortex, and left rolandic operculum (OP1).

The contrast Imagery(R) - Observation indicated activation in the left SMA (6) and left inferior frontal gyrus (44). Imagery(R) - PassiveMove showed activation in the left anterior precentral gyrus (6).

Taken together, $\operatorname{Imagery}(\mathrm{R})$ is predominantly associated with premotor and prefrontal areas, but only moderately with the primary motor and somatosensory areas observed in Execution.

\subsubsection{Observation}

Contrasting Observation - Base $(\mathrm{O})$ showed very circumscribed foci of sensorimotor activation in the left and right precentral gyri (6) and in the left inferior parietal lobe (2). Calculating the contrast Observation - Execution revealed an activation in the right precentral gyrus (4a). In addition, a number of prefrontal areas in the inferior, middle, and superior frontal gyri, partially extending into the anterior inferior precentral gyrus, were activated. The reversed contrast, i.e. Execution - Observation, resulted in virtually the same activation pattern as compared to Execution - Base $(C)$, i.e. activation in the left sensorimotor cortices (pre- and postcentral gyri, rolandic operculum, 4a, 6, 1, 2, OP1), and bilateral SMA (6).

The contrasts Observation - Imagery(R) and Observation - PassiveMove both showed extended activation of posterior visual areas and of scattered prefrontal areas, but no activation of somatosensory, motor, or premotor areas.

Taken together, there is rather circumscribed activation of the sensorimotor cortices in Observation.

\section{9 fMRI Results Patients}

Please note that all patients had right-hemispheric lesions. Since we aimed at assessing the affected hand, all tasks were performed with the left hand. Accordingly, activation patterns might be left-right reversed as compared to the HVs. Results of baseline comparisons are presented in Figure 4 and Table 4, for a figure of all pairwise comparisons see supplementary online materials.

During evaluation of results we noticed that PassiveMove showed a strong deactivation of posterior brain areas (see Discussion for potential reasons). These deactivations appeared as activations in contrasts in which PassiveMove is subtracted, e.g. Imagery - PassiveMove. However, since these activations are not genuinely due to the compared condition (Imagery in this example), we controlled for them by masking $(\mathrm{p}(\mathrm{FWE})<.05)$ the comparison with the condition specific network (Imagery - Base(C) in this example). The assumption is that only areas showing activity when compared to a low-level resting baseline need to be considered for comparisons of the respective condition with PassiveMove, since all other areas can only be due to deactivations in PassiveMove.

\subsubsection{Execution}

Although patients were instructed to perform the same wrist movement as HVs, no patient was able to do so. As described in detail above actual movement during Execution was virtually absent or, at best, much slower and with very small amplitude. Therefore, in patients the Execution condition may be better understood as the attempt to execute overt movement.

Calculating the contrast Execution - Base(C) showed bilateral activation in the lateral and medial motor and premotor cortices. In particular, left, right and medial primary motor cortices in the pre- and postcentral gyri (4a, 4p, medial 4a) and the left rolandic operculum (44, OP4) were involved in attempted Execution. The latter further activated bilateral premotor cortices in the precentral gyri $(6$, medial 6/SMA) extending into the right superior (6) and left inferior frontal (44) gyri. Posteriorly, the activation extended into the somatosensory cortices of the left and right postcentral gyri (2). It is noteworthy that the consistency of these sensorimotor activations across patients is high. As can be seen in Table 4, for virtually all activation peaks all five patients showed the expected effect (i.e., positive beta-value) in close vicinity of or even directly at the peak voxel of the group statistics. Taken together, Execution activated bilateral and medial sensorimotor cortices.

\subsubsection{Passive Movement}

The contrast PassiveMove - Base (C) showed an extended activation in the right sensorimotor cortex including pre- and postcentral gyri. While the focus of the activation was on somatosensory areas $(1,2,3 b)$, it also extended anteriorly into motor areas $(4 \mathrm{a}, 4 \mathrm{p})$. Medially, SMA (6) was activated. Calculating the contrast PassiveMove - Execution (I) revealed no sensorimotor activations. The reversed contrast, i.e. Execution - Passive Move (I) showed extended activations in the left sensorimotor cortices, i.e. precentral gyrus (6), SMA (6), paracentral lobule (6, $4 a)$, postcentral gyrus $(1,2,3 b)$, and rolandic operculum (OP3). Sensorimotor cortices in the right hemisphere were activated less intense, and in particular lateral primary motor activation was absent.

The contrast PassiveMove - Imagery(L) revealed an extended activation in the right somatosensory cortex $(2,3 b, 1)$ extending into motor $(4 a, 4 p)$ and parietal (7PC, 7A, hIP2) areas. PassiveMove Observation showed a highly similar pattern, with an extended activation in the right somatosensory cortex $(1,3 b, 2)$ extending into motor $(4 a, 4 p)$ and parietal (7PC) areas.

Taken together, PassiveMove mainly activated right somatosensory cortices and, to a lesser extent, primary motor areas. This activation was stronger in PassiveMove than in Observation and Imagery, but not attempted Execution. Instead, PassiveMove showed weaker activation than attempted Execution in left and, to a lesser extent, right somatosensory cortices. 


\subsubsection{Imagery $(L)$}

Contrasting Imagery $(\mathrm{L})-\operatorname{Base}(\mathrm{C})$ revealed a network of sensorimotor areas comparable to Execution - Base $(C)$. In particular, premotor $(6,44)$ and primary motor areas (4a) in the precentral gyrus and somatosensory areas in the postcentral gyri (3b) were activated bilaterally. The activations extended anteriorly into the inferior frontal gyrus $(44,45)$ and posteriorly into the superior parietal lobe (2). Medially, SMA and paracentral lobule $(6,4 a)$ were activated. Notably, a number of activation peaks were localized virtually identical to those obtained in the Execution - Baseline contrast, and the consistency of the activations across patients was again very high.

The contrast Imagery(L) - Execution again revealed no sensorimotor activation. The reversed contrast Execution - Imagery (L) showed sensorimotor activation in the right postcentral gyrus, extending into the precentral gyrus $(1,3 b, 4 p, 4 a, 6)$.

Imagery - Observation showed activation in the right sensorimotor cortex of the postcentral gyrus (3b, 4a) and the right SMA (6). In the contrast Imagery PassiveMove (masked by Imagery - Baseline) the precentral gyrus was activated in the left $(4 a, 6)$ and right (6) hemisphere, with activation of both extending into the postcentral gyri $(1,3 b)$. Lateral activations were further evident in the left inferior frontal gyrus (44), and left rolandic operculum (OP4). Medially, left SMA $(4 a, 6)$ and paracentral lobule $(6,4 a)$ were activated.

Taken together, when compared to $\operatorname{Base}(\mathrm{C})$, Imagery $(\mathrm{L})$ showed a pattern highly similar to Execution, i.e. bilateral and medial sensorimotor cortices. This activation was stronger in the right hemisphere, and also evident when Imagery(L) was compared to PassiveMove and Observation, but not when compared to Execution. It is interesting to note that the sensorimotor activation of Imagery $(\mathrm{L})$ is slightly anterior to the more somatosensory activation of Passive Movement. Attempted Execution activates right sensorimotor cortices stronger than Imagery(L).

\subsubsection{Observation}

For the contrast Observation - Base $(\mathrm{O})$, activation of the sensorimotor system was confined to the right lateral and medial anterior premotor areas (6) and a small focus in the right inferior parietal lobe (2). The contrast Observation - Execution revealed no sensorimotor activation. The reversed contrast Execution - Observation showed sensorimotor activation in the right postcentral gyrus $(1,4 \mathrm{a})$ and left precentral (6) and postcentral (1) gyri. In addition, the paracentral lobule $(4 a, 6)$, the left rolandic operculum (OP3), and left inferior frontal gyrus (44) were activated.

The contrast Observation - Imagery $(\mathrm{L})$ revealed sensorimotor activation limited to anterior parts of the right premotor cortices (superior frontal gyrus, 6; precentral gyrus, 6) and medially to the paracentral lobule (SMA, 6). In Observation - PassiveMove (masked by Observation - Baseline) sensorimotor activation was evident in right hemispheric premotor areas (middle frontal gyrus (6), rolandic operculum $(44,6)$, precentral gyrus $(6,4 a)$ and medially in the left SMA (6) and paracentral lobule (6).

Taken together, Observation was characterized by mainly rather anteriorly located premotor activations, but only moderately by primary motor or somatosensory activations.

\section{Discussion}

\subsection{Summary of findings}

In healthy volunteers (HVs) overt execution of wrist movements activated a network of areas in left lateral sensorimotor cortices, medial motor cortices, and parietal areas. The condition mirroring this pattern most closely is PassiveMove, which activated a virtually identical network. Like Execution, Imagery(R) also activated the SMA. However, instead of left lateralized more primary sensorimotor cortices, Imagery(R) activated bilateral lateral premotor and prefrontal areas. Thus, while Execution activates more primary cortices associated with motor execution, Imagery(R) activates predominantly premotor and prefrontal areas associated with motor planning. Observation, finally, showed only few circumscribed activations in sensorimotor cortices, thus bearing the least resemblance with Execution.

In patients, the attempt to execute wrist movements extensively activated the sensorimotor system, including primary motor and sensory areas. This is remarkable given the virtually absent movement abilities. In contrast to healthy volunteers, the condition most closely mirroring this pattern is Imagery $(\mathrm{L})$. The condition mirroring the pattern of Execution second best is PassiveMove, which was characterized by an extended activation of the right (contralateral) primary sensorimotor cortices (remember that patients performed the task with the left hand). Differences between PassiveMove and Execution occurred mainly in left sensorimotor cortices and in more anterior and more posterior right hemispheric areas. Finally, Observation resembled Execution least and was characterized by lateral and medial premotor activations.

\subsection{Adherence to the instruction}

An important aspect when discussing the findings is the participant's adherence to the instructions. Since motor imagery, movement observation, and passive movement involve merely internal processes, objective measures are hard to obtain. Generally, two basic patterns of non-adherence are conceivable. First, participants may just not perform the task. While such behavior would be obvious in motor execution, it may be critical if participants do not perform imagery or do not pay attention to the video. In line with previous reports (Bardin, Fins et al. 2011), our data speak against this possibility, since all participants showed at least some sensorimotor activation during all tasks. Second, participants may perform the wrong task, or may not follow the instruction correctly. In particular, 
it may be critical when participants actively move their wrist during PassiveMove, imagery, or observation. While we tried to avoid such influences, e.g. by measuring forces exerted by the hand and by visual inspection, we cannot conclusively exclude such behavior. Unfortunately, electromyography as a potentially more sensitive measure (but see Guillot and Collet 2010) was not available to us. However, generally force data and visual inspection confirm that, if at all, only subthreshold muscular activity might have been present.

While adhering to the instruction is important for gaining theoretical knowledge, it may be less relevant for clinical application. For instance, some $\mathrm{HVs}$ reported that it was difficult in PassiveMove to not help the movement, i.e. staying purely passive. Thus, although we took measures to avoid this situation, we cannot exclude conclusively that HVs did move actively to some degree during PassiveMove. While this may affect the conclusions about the functional neuroanatomical correlates of PassiveMove in an undesired way, it may actually be a beneficial (and therefore desired) behavior for the patients. If the tendency to help the movement is present in patients as well, PassiveMove may be a good rehabilitation method because it automatically triggers the attempt to move overtly. In other words, for the therapeutic intervention the outcome is of importance, not whether patients really control their wish to help the movement.

Therefore, we suggest to view our results from two perspectives: First, the scientist's perspective, scrutinizing which mental processes may be the true cause of an activation; Second, the practitioner's perspective, taking the results as they are with the only interest in which instruction leads to which activation pattern irrespective of what the underlying mental processes truly are.

\subsection{Motor execution}

In HVs, overt movement of the right wrist recruited a network consisting mainly of left pre- and postcentral gyrus, left SMA, bilateral supramarginal gyri, and right cerebellum. These areas are involved in motor control in general (Roland 1984; Stephan, Fink et al. 1995; Gerardin, Sirigu et al. 2000) and in posture control of the wrist in particular (Suminski, Rao et al. 2007). In patients, the attempt to overtly move the left (paretic) wrist recruited the same areas plus a number of additional regions. These included in particular more anterior premotor and prefrontal cortices and more extended parietal cortices. Generally, activation was more bilateral in patients, including activation of the ipsilateral pre- and primary motor cortices. The additional recruitment of areas beyond the ones observed in HVs is a typical finding for patients with low recovery (Ward, Brown et al. 2003; Lehericy, Gerardin et al. 2004; Kimberley, Khandekar et al. 2006). These results demonstrate that already the attempt to move a limb, even if only minimal movement is actually possible, activates the motor system of the brain. Whether these results hold for brain damaged patients with a full loss of motor control remains to be tested. Promising evidence comes from patients suffering from complete spinal cord injury who despite their chronic state still demonstrate motor system activation in response to attempted overt movement (Cramer, Lastra et al. 2005; Hotz-Boendermaker, Funk et al. 2008).

\subsection{Motor Imagery}

The cortical network activated by motor imagery in patients as well as HVs is well in line with previous reports (Grèzes and Decety 2001; Lotze and Halsband 2006). While lateral and medial premotor cortices were involved in motor imagery in both groups, only patients showed (bilateral) primary motor activation. Mainly due to this difference, motor imagery resembles motor execution best in patients, but only second-best in HVs. While the reasons for this difference are unclear, it is interesting to note that while the involvement of the primary motor cortex in motor imagery is highly variable and debated in HVs (Jackson, Lafleur et al. 2001; Dechent, Merboldt et al. 2004; Sharma, Pomeroy et al. 2006; Sharma, Jones et al. 2008), patient studies seem to show primary motor cortex involvement more consistently (Lehericy, Gerardin et al. 2004; Kimberley, Khandekar et al. 2006; Sharma, Baron et al. 2009; Sharma, Simmons et al. 2009). A reason for this may be that motor imagery is more difficult for patients so that additional neural resources are recruited for task performance. However, most previous studies in healthy subjects showed that neither movement complexity (as an operationalization for movement difficulty and task demands) nor motor imagery proficiency affect the neuroanatomical correlates of MI (Gerardin, Sirigu et al. 2000; Boecker, Ceballos-Baumann et al. 2002; Lehericy, Gerardin et al. 2004; Guillot, Collet et al. 2008) (but see Kuhtz-Buschbeck, Mahnkopf et al. 2003). Thus, an alternative explanation would be that the extended activations in patients as compared to HVs mimic the pattern observed for overt attempted execution. The lesion is likely to have caused a reorganization of the motor system (Ward, Brown et al. 2003) which also may have changed the functional neuroanatomical correlates of motor imagery (Sharma, Simmons et al. 2009).

Taken together, the results on motor imagery allow for two conclusions. First, in patients suffering from severe motor deficits after stroke motor imagery is a very good approximation of overt movement and actually activates primary motor cortices. Second, care has to be taken if one aims at transferring the results gained by $\mathrm{HVs}$ to patients since the functional neuroanatomical correlates of motor imagery differed between the groups (see also (Stinear, Fleming et al. 2007)).

\subsection{Passive Movement}

In HVs as well as patients the activation pattern during PassiveMove was highly similar to overt movement in HVs. With respect to sensorimotor activation, mainly the contralateral sensorimotor 
cortices as well as the SMA were activated. Compared to the patients, activation in the HVs extended more anteriorly into the precentral gyrus and also included inferior parietal areas. Generally, these activation patterns are in line with previous evidence on the functional neuroanatomical correlates of passive movement (Weiller, Juptner et al. 1996; Naito, Ehrsson et al. 1999; Naito, Roland et al. 2002; Ciccarelli, Toosy et al. 2005; Guzzetta, Staudt et al. 2007) and the assumption that the afferent feedback produced by passive movement not only targets somatosensory but also motor areas (Fetz, Finocchio et al. 1980; Lemon 1999; Dechaumont-Palacin, Marque et al. 2008).

In HVs PassiveMove was the condition most similar to overt execution, while there were considerable differences in patients. In more detail, in patients sensorimotor activation was strictly contralateral in PassiveMove while it was bilateral in attempted execution, and the medial (pre)motor activation in the area of the SMA was considerably smaller in PassiveMove than in overt execution. This pattern of results suggests that PassiveMove activates the motor system in a rather passive and/or automatic way which is, if at all, only moderately affected by reorganization due to brain damage. In attempted execution and imagery the patients try to implement a motor program (Hanakawa, Dimyan et al. 2008). Such more complex planning processes may be affected by the lesion so that for their fulfillment other (mental and/or neural) strategies relying on further brain areas are required. Activations during PassiveMove, on the other hand, may be driven purely by afferent sensory input (Weiller, Juptner et al. 1996; Mima, Sadato et al. 1999). If this input is affected by the lesion, there is no behavioral need for other strategies, since there is no task or behavioral goal like production of a movement. Consequently, passive movement in patients is comparable to overt and passive movement in HVs, but less comparable to their own attempted execution or imagery. A somewhat related, non-exclusive, explanation of these findings may be that generally greater functional reorganization takes places in the efferent system than the afferent system.

Three conclusions can be derived from these arguments. First, if the goal is to induce motor system activation in patients as similar as possible as in HVs, PassiveMove is the best choice. Second, if the goal is to activate the full motor system as evident in attempted Execution, i.e. including areas involved probably due to neural reorganization, PassiveMove seems inferior to Imagery. Third, at least in our paradigm the findings for PassiveMove in HVs are transferrable to patients.

The results obtained for patients hold potentially interesting implications for treatment interventions. For example, we found that patients activate a wider neural network, including e.g. ipsilateral motor cortices, than HVs when attempting to execute or imagine an affected arm movement. On the one hand, such wider recruitment has been linked to patients with low recovery. On the other hand, however, it has been suggested that the additional neural activation may support planning and executing the movement, i.e. it is functionally relevant and not an epiphenomenon (Ward, Brown et al. 2003; Tombari, Loubinoux et al. 2004; Ward, Brown et al. 2006). The present study supports the latter assumption that the additional areas recruited for attempted execution and imagery form an integral part of the reorganized motor system in persons with low-functioning chronic hemiparesis. Moreover, Nelles et al. (1999) have found that motor recovery after stroke was associated with increasing activation of ipsilateral premotor areas. If we assume that recruitment of the ipsilateral premotor cortex for affected arm control supports the recovery of function, a covert training method which activates the reorganized motor system is required. The latter is achieved by imagery and attempted execution but not through passive movement. We therefore predict that an imagery-based intervention is more successful in patients with poor residual ability than a passive movement based intervention. Critically, contemporary physical therapy often relies on passively moving the limb in the early stages of recovery which may not be the best way of helping patients to regain function. However, this has to be conclusively demonstrated by studies directly comparing treatment success of the different approaches.

\subsection{Observation}

In HVs, observation caused only weak activations in the sensorimotor system which were restricted to bilateral premotor cortices and the left IPL. Activation of primary sensorimotor areas was not evident. In patients, observation activated a set of similar areas with the exception that premotor and IPL activation was restricted to the right hemisphere and that additionally the SMA was activated. Thus, in HVs as well as patients observation resembled execution to the least.

Generally, the activation patterns observed in the present study are in line with previous research (Caspers, Zilles et al. 2010). While frequently ventral premotor cortices have been associated with movement observation (Decety and Grèzes 1999), the presently observed more dorsal premotor activations have been reported before particularly for the observation of wrist and hand movements (Buccino, Binkofski et al. 2001; Sakreida, Schubotz et al. 2005; Morin and Grezes 2008). Interestingly, the presently observed areas were part of a network showing increased activation in response to rehabilitation using action observation (Ertelt, Small et al. 2007). The absence of more primary sensorimotor activations is in line with early reports (Decety, Perani et al. 1994) and may be explained by the chosen task instruction.

In more detail, previous research indicated that activation evoked by movement observation may potentially be reduced when the movement is simple (Biagi, Cioni et al. 2010), does not involve objects (Buccino, Binkofski et al. 2001; Morin and Grezes 2008), and when the instruction does not stress that 
the movements are supposed to be imitated after having been watched (Frey and Gerry 2006) (but see Caspers, Zilles et al. 2010). Thus, by using other actions and instructions we may have had observed stronger activation of the sensorimotor system, potentially resulting in a better resemblance of Execution. However, we chose the current movement and task deliberately for the following reason. Bearing clinical practice in mind, it seems problematic to present patients who lost all or virtually all their motor abilities in the affected limb with a video of a rather complex movement, such as grasping an object, and ask them that they should overtly repeat the movement afterwards. This may overwhelm patients so that they do not follow the instruction and do not cooperate in this task. To avoid this, we chose a more realistic scenario, namely that severely paretic patients will initially try to produce a very basic and simple movement, such as a wrist flexion-extension (cf. Celnik, Webster et al. 2008) (but see Pomeroy, Clark et al. 2005). At the same time we also ensured that the same movement was used in all conditions (for instance, grasping an object is difficult to realize as a passive movement). Thus, we conclude that by modifying the paradigm observation may better resemble execution (scientist's perspective) but at the cost of less practicability for the clinical application (practitioner's perspective).

It is noteworthy that in patients activation of the sensorimotor system is strongly right (contralateral) lateralized, as already observed for PassiveMove. Thus, the same arguments as for PassiveMove hold here as well, i.e. that Observation as a purely passive task without a behavioral goal to move or imagine to move may not fully recruit the reorganized motor system as identified by attempted Execution. In particular, ipsilateral sensorimotor areas, which may be functionally relevant for movement planning and execution after neural reorganization, are not activated. Consequently, in patients Observation is roughly comparable to Observation in $\mathrm{HVs}$, but it shows marked differences to their own attempted Execution. However, future research should test whether this pattern holds with more powerful observation paradigms.

Taken together, it seems that when using movements appropriate for therapeutic interventions in very low functioning patients movement observation may be less well suited than in particular motor imagery.

\subsection{Comparison of movement modes}

The discussion so far mainly dealt with the question how well the different covert movement modes resemble overt execution. This question was motivated from a clinical perspective, as we followed the suggestion that motor system activation is beneficial for motor recovery (Johnson-Frey 2004; Sharma, Pomeroy et al. 2006; Munzert, Lorey et al. 2009). In this section, we will focus more on the scientist's perspective and scrutinize in more detail the potential underlying processes giving rise to the observed activation patterns.

Our description of the processes involved in the different movement modalities is based on Gazzola and Keysers' (2009) suggestion that overt execution of a movement involves the planning and programming of the movement (premotor cortex), the actual control of muscles (primary motor cortex), and the feeling of the own movement (somatosensory cortex). Because the movement was simple (wrist flexion-extension), demands on complex planning were rather low and, consequently, lateral premotor cortices were only moderately activated. In more detail, when thresholded at $\mathrm{p}<.05$ (FWE) lateral premotor activations were evident only as the main primary motor activation slightly extending into premotor areas (Fig. 1A). However, using a reduced threshold of $\mathrm{p}<.001$ (uncorrected), Execution showed additional premotor activation foci, which were similar to Imagery. This subthreshold activation in Execution explains the absence of statistically significant differences in premotor activations between Imagery and Execution (Supplementary Figure 2, panel 3b). The medial premotor cortices (SMA) are probably involved in either the performance of a rhythmic movement (Shima and Tanji 2000) or the generation of a go-signal for overt motor output (Gazzola and Keysers 2009).

It is assumed that motor imagery is an internal simulation of the overt movement program (Jeannerod 1994; Jeannerod and Frak 1999). Thus, the essential process to perform motor imagery is the programming and planning of the movement in premotor cortices. Consequently, we observed lateral and medial premotor activation during Imagery. These areas were activated during Execution only at a reduced threshold ( $\mathrm{p}<.001$, uncorrected) and failed to reach statistical significance in the direct comparison Imagery Execution. The tentatively higher activation during Imagery than Execution might be explained by the fact that the generation of a vivid kinesthetic motor image probably requires more effort and may involve more explicit motor planning than the overt performance of the simple wrist movement. An additional demand, not present in overt execution, is to inhibit the overt execution of the programmed movement. It has been suggested that the SMA plays a gatekeeper role for this, for instance by inhibiting primary motor cortex activity or the connectivity between pre- and primary motor cortices (Chen, Yang et al. 2009; Gazzola and Keysers 2009). This gatekeeping, however, is probably not perfect because primary motor cortex activation is occasionally observed during motor imagery (Lotze, Montoya et al. 1999). In addition, studies using other measures, such as TMS, showed that during (in particular kinesthetic) motor imagery motor facilitation in primary motor cortices can still be observed (Stinear, Byblow et al. 2006). Thus, although the SMA activation may appear comparable in Execution and Imagery, the observed activation may serve different functional purposes (Gazzola and Keysers 2009). Finally, the absence of 
overt movement, which in turn results in the absence of sensory feedback, may well explain the absence of activation in primary motor cortex and somatosensory cortices.

For passive movement no processes are essentially required, since there is neither an overt nor a covert motor task. However, peripheral sensory afferent signals are present, which have been shown to target not only sensory but also motor areas. Consequently, we argue that the observed activations in the primary motor and somatosensory cortices (which were lower than in Execution but higher than Imagery, cf. Supplementary Fig. 1) are due to the somatosensory feedback of the passive movement.

For Observation again no processes are essentially required, since there is neither an overt nor a covert motor task. In addition, due to lack of overt movement there is also no sensory feedback. However, it is known that the mirror neuron system is active already during the mere observation of movements. Gazzola and Keysers (2009) argued that such activation may arise because the motor system is able to specify actions by their anticipated sensory consequences (inverse models of motor control, Wolpert and Kawato 1998). The observation of a movement may activate these representations (probably in middle temporal and inferior parietal areas), and the activation may spread to tightly interconnected areas such as the inferior premotor cortex. Thus, the observed action activates the premotor representation of that same action as if the participant is planning to perform the action. Once these representations are activated they may be employed to predict the actions of others (by use of forward models of motor control, Wolpert and Kawato 1998). This indirect flow of activations among areas may involve further areas, such as more primary motor and somatosensory cortices, and indications for mirror neurons in these areas have been reported in humans (Gazzola and Keysers 2009). However, our Observation paradigm resulted only in weak activation of the mirror neuron system. The main reason for this is probably that our paradigm did not require any active processing of the observed movements. If, instead, participants are asked to repeat the observed movement later, much deeper and more active processing may occur (Frey and Gerry 2006). In such a case, our initial statement that Observation does not require any processes might have to be revised, because active processing and memorizing a movement imposes demands on the motor system beyond the passive activation induced by the observation condition in our study. Taken together, there was only very weak sensorimotor activation in Observation in our particular paradigm due to the absence of any motor task or overt behavior. Sensorimotor activation may be increased by requiring the participants to actively process the observed movements in some way. As argued above, this, however, may decrease the applicability of Observation in the rehabilitation of severely affected patients.
Finally, one may speculate about differences in the involved processes between patients and HVs. Regarding overt Execution, patients showed a higher number and more extended activation clusters, in particular in bilateral premotor and ipsilateral sensorimotor areas. There are at least two explanations for these differences, that is, effort and reorganization. First, one may argue that the task required much more effort for patients than for HVs, which may have resulted in the more extended activation pattern. However, previous research showed that increased effort is mainly associated with a spatial extension of the activation of already activated areas, but neither with a recruitment of new, previously not activated areas, nor with an increase in activation amplitude (Thickbroom, Phillips et al. 1998). In addition, the lateralization pattern does not change with increased effort (Archer, Abbott et al. 2004). Thus, increased effort in patients as compared to HVs may explain more extended contralateral primary motor cortex activation, but not the presently observed additional activations in ipsilateral primary and premotor areas and contralateral premotor areas. Consequently, we interpret the differences between patients and HVs as the result of the second possible alternative, i.e. the reorganization of the motor system in response to the brain damage (Ward 2004).

In this context, one may ask about the relationship between the learning of new motor skills in healthy participants and the relearning of motor skills in patients suffering from motor deficits. For the learning of new skills in healthy participants, complex patterns of activation increases and decreases in several brain areas have consistently been reported (Dayan and Cohen 2011; Krakauer and Mazzoni 2011; Penhune and Steele 2012). However, the neural correlates of motor skill relearning in patients are less well understood, so that at present it remains unclear whether motor (re-)learning in healthy participants and patients rely on similar or different mechanisms (Hosp and Luft 2011).

Motor imagery might have differed between both groups, because HVs overtly performed the wrist movement throughout the experiment which may have facilitated a vivid motor image. Patients, on the other hand, performed overt execution (each patient was able to produce an overt wrist movement at least once, see Table 2 and Supplementary Table 2) only after the other conditions had been acquired, so that the motor image was probably based on movements longer ago, maybe even from pre-stroke times. This may have resulted in a less vivid motor image, which may have resulted in lower activation levels (Lorey, Pilgramm et al. 2011). However, it is noteworthy that Imagery was nevertheless the covert movement mode which resembled attempted execution best. Thus, by trying to increase the vividness of the motor image, e.g. by passive movement or attempted movement performed before the motor imagery, even stronger activations may be observed during Imagery.

Since passive movement and observation were purely passive tasks, there should be no differences in 
the involved processes between $\mathrm{HVs}$ and patients, which is in line with our finding of equivalent activation patterns between both groups. However, if more active paradigms are used (e.g. Observation with the goal to imitate later), differences between patients and HVs may arise, because such active processing may employ the reorganized motor system in patients, which differs from the motor system in HVs.

\subsection{Limitations}

There are some limitations to be considered. First, the two investigated groups are no matched controls but instead independent samples (healthy student volunteers vs. elderly stroke patients). Consequently, caution is advised when the groups are compared. Second, the patient group consisting of five participants is rather small so that we had to use a fixed-effect model for statistical evaluation of the fMRI data. In a strict statistical sense the present patient data, therefore, cannot be generalized to the population. However, the high consistency of observed activation peaks is promising that future studies with larger samples are likely to replicate the present findings. Third, the difference in sample size prevented statistical comparisons between groups so that the reported group differences are only descriptive. Fourth, subthreshold muscular activity cannot be excluded since we had no measures such as EMG available.

There was an unanticipated finding in the PassiveMove condition in patients, which showed a strong deactivation of posterior brain areas. The reason for this deactivation, which spared the right somatosensory cortex, is unclear but may be caused by the patients intensely focusing on the sensation of the passively moving affected hand. This may have deducted attention from other mental activities, resulting in reduced activity in the associated areas. A comparable pattern has been reported for the default network which is usually active only during resting periods but less active during task periods, resulting in the often observed de-activations when task conditions are compared to resting baseline conditions (Gusnard and Raichle 2001).

\subsection{Conclusion}

In the present study we investigated the three major approaches of stimulating the motor system without overt movement, i.e. motor imagery, passive movement, and movement observation, together with overt execution as a repeated measures design in healthy volunteers and a pilot sample of five patients suffering from chronic severe hemiparesis after stroke. Our results are highly promising in that all covert movement approaches activate the sensorimotor cortices. However, profound differences were found between the different approaches and investigated samples. In healthy volunteers, overt Execution was best resembled by PassiveMove, closely followed by Imagery, and only moderately well by Observation. In patients, attempted overt Execution demonstrated the functional reorganization of the sensorimotor system.
Imagery most closely mirrored the activation patter driven by attempted execution followed by PassiveMove. The overlap of neural activations between attempted Execution and Observation was only moderate. We conclude that Imagery seems the most promising approach to activate the motor system in hemiparetic stroke patients not in the least because it recruits the reorganized sensorimotor system rather than typical activations observed for Execution in healthy controls. Our data further suggest to exercise caution when transferring results between patient samples and healthy volunteers. 


\section{References}

Andersson, J. L., C. Hutton, et al. (2001). "Modeling geometric deformations in EPI time series." Neuroimage 13(5): 903-919.

Archer, J. S., D. F. Abbott, et al. (2004). "Stable ratio of ipsilateral to contralateral sensory motor cortex activity despite varying effort and peripheral nerve block." J Clin Neurosci 11(1): 46-52.

Bardin, J. C., J. J. Fins, et al. (2011). "Dissociations between behavioural and functional magnetic resonance imaging-based evaluations of cognitive function after brain injury." Brain 134(Pt 3): 769-782.

Biagi, L., G. Cioni, et al. (2010). "Anterior intraparietal cortex codes complexity of observed hand movements." Brain Res Bull 81(4-5): 434-440.

Boecker, H., A. O. Ceballos-Baumann, et al. (2002). "A H(2)(15)O positron emission tomography study on mental imagery of movement sequences--the effect of modulating sequence length and direction." Neuroimage 17(2): 999-1009.

Braun, S. M., A. J. Beurskens, et al. (2006). "The effects of mental practice in stroke rehabilitation: a systematic review." Arch Phys Med Rehabil 87(6): 842-852.

Brett, M., J.-L. Anton, et al. (2002). "Region of interest analysis using an SPM toolbox." $\underline{\text { 8th }}$ International Conference on Functional Mapping of the Human Brain, June 2-6, Sendai, Japan(Available on CD-ROM in NeuroImage, Vol 16, No 2).

Buccino, G., F. Binkofski, et al. (2001). "Action observation activates premotor and parietal areas in a somatotopic manner: an fMRI study." Eur J Neurosci 13(2): 400-404.

Carel, C., I. Loubinoux, et al. (2000). "Neural substrate for the effects of passive training on sensorimotor cortical representation: a study with functional magnetic resonance imaging in healthy subjects." J Cereb Blood Flow Metab 20(3): 478-484.

Caspers, S., K. Zilles, et al. (2010). "ALE metaanalysis of action observation and imitation in the human brain." Neuroimage 50(3): 1148-1167.

Celnik, P., B. Webster, et al. (2008). "Effects of action observation on physical training after stroke." $\underline{\text { Stroke }}$ 39(6): 1814-1820.

Chen, H., Q. Yang, et al. (2009). "Evaluation of the effective connectivity of supplementary motor areas during motor imagery using Granger causality mapping." Neuroimage 47(4): 1844-1853.
Ciccarelli, O., A. T. Toosy, et al. (2005). "Identifying brain regions for integrative sensorimotor processing with ankle movements." Exp Brain Res 166(1): 31-42.

Clark, S., F. Tremblay, et al. (2003). "Differential modulation of corticospinal excitability during observation, mental imagery and imitation of hand actions." Neuropsychologia 42(1): 105-112.

Cramer, S. C., L. Lastra, et al. (2005). "Brain motor system function after chronic, complete spinal cord injury." Brain 128(Pt 12): 2941-2950.

Cramer, S. C., E. L. Orr, et al. (2007). "Effects of motor imagery training after chronic, complete spinal cord injury." Exp Brain Res 177(2): 233-242.

Dayan, E. and L. G. Cohen (2011). "Neuroplasticity subserving motor skill learning." Neuron 72(3): 443454.

Decety, J. (1996). "Do imagined and executed actions share the same neural substrate?" Brain Res Cogn Brain Res 3(2): 87-93.

Decety, J. and J. Grèzes (1999). "Neural mechanisms subserving the perception of human actions." Trends Cogn Sci 3(5): 172-178.

Decety, J. and M. Jeannerod (1995). "Mentally simulated movements in virtual reality: does Fitts's law hold in motor imagery?" Behav Brain Res 72(12): 127-134.

Decety, J., D. Perani, et al. (1994). "Mapping motor representations with positron emission tomography." Nature 371(6498): 600-602.

Dechaumont-Palacin, S., P. Marque, et al. (2008). "Neural correlates of proprioceptive integration in the contralesional hemisphere of very impaired patients shortly after a subcortical stroke: an FMRI study." Neurorehabil Neural Repair 22(2): 154-165.

Dechent, P., K. D. Merboldt, et al. (2004). "Is the human primary motor cortex involved in motor imagery?" Brain Res Cogn Brain Res 19(2): 138-144.

Eickhoff, S. B., K. E. Stephan, et al. (2005). "A new SPM toolbox for combining probabilistic cytoarchitectonic maps and functional imaging data." Neuroimage 25(4): 1325-1335.

Ertelt, D., S. Small, et al. (2007). "Action observation has a positive impact on rehabilitation of motor deficits after stroke." Neuroimage 36 Suppl 2: T164173.

Feltz, D. H. and D. M. Landers (1983). "The effects of mental practice on motor skill learning and 
performance: a meta-analysis." Journal of Sport Psychology 5: 25-57.

Fetz, E. E., D. V. Finocchio, et al. (1980). "Sensory and motor responses of precentral cortex cells during comparable passive and active joint movements." J Neurophysiol 43(4): 1070-1089.

Filimon, F., J. D. Nelson, et al. (2007). "Human cortical representations for reaching: mirror neurons for execution, observation, and imagery." Neuroimage 37(4): 1315-1328.

Frey, S. H. and V. E. Gerry (2006). "Modulation of neural activity during observational learning of actions and their sequential orders." J Neurosci 26(51): 13194-13201.

Friston, K. J., A. P. Holmes, et al. (1999). "Multisubject fMRI studies and conjunction analyses." NeuroImage 10(4): 385-396.

Friston, K. J., A. P. Holmes, et al. (1995). "Statistical Parametric Maps in Functional Imaging: A General Linear Approach." Human Brain Mapping 2: 189-210.

Garrison, K. A., C. J. Winstein, et al. (2010). "The mirror neuron system: a neural substrate for methods in stroke rehabilitation." Neurorehabil Neural Repair 24(5): 404-412.

Gazzola, V. and C. Keysers (2009). "The observation and execution of actions share motor and somatosensory voxels in all tested subjects: singlesubject analyses of unsmoothed fMRI data." $\underline{\text { Cereb }}$ Cortex 19(6): 1239-1255.

Gerardin, E., A. Sirigu, et al. (2000). "Partially overlapping neural networks for real and imagined hand movements." Cereb Cortex 10(11): 1093-1104.

Grèzes, J. and J. Decety (2001). "Functional anatomy of execution, mental simulation, observation, and verb generation of actions: a meta-analysis." Human Brain Mapping 12(1): 1-19.

Guillot, A. and C. Collet (2010). The neurophysiological foundations of mental and motor imagery. Oxford, UK, Oxford University Press.

Guillot, A., C. Collet, et al. (2008). "Functional neuroanatomical networks associated with expertise in motor imagery." Neuroimage 41(4): 1471-1483.

Guillot, A., C. Collet, et al. (2009). "Brain activity during visual versus kinesthetic imagery: an fMRI study." Hum Brain Mapp 30(7): 2157-2172.

Gusnard, D. A. and M. E. Raichle (2001). "Searching for a baseline: Functional imaging and the resting human brain." Nature Reviews Neuroscience 2(10): 685-694.

Guzzetta, A., M. Staudt, et al. (2007). "Brain representation of active and passive hand movements in children." Pediatr Res 61(4): 485-490.

Hanakawa, T., M. A. Dimyan, et al. (2008). "Motor planning, imagery, and execution in the distributed motor network: a time-course study with functional MRI." Cereb Cortex 18(12): 2775-2788.

Hesse, S., C. Bertelt, et al. (1995). "Treadmill training with partial body weight support compared with physiotherapy in nonambulatory hemiparetic patients." Stroke 26(6): 976-981.

Hosp, J. A. and A. R. Luft (2011). "Cortical plasticity during motor learning and recovery after ischemic stroke." Neural Plast 2011: 871296.

Hotz-Boendermaker, S., M. Funk, et al. (2008). "Preservation of motor programs in paraplegics as demonstrated by attempted and imagined foot movements." Neuroimage 39(1): 383-394.

Hou, W., S. Shen, et al. (2005). "An MRI compatible visual force-feedback system for the study of force control mechanics." Conf Proc IEEE Eng Med Biol Soc 4: 3687-3690.

Iacoboni, M. and J. C. Mazziotta (2007). "Mirror neuron system: basic findings and clinical applications." Ann Neurol 62(3): 213-218.

Iseki, K., T. Hanakawa, et al. (2008). "Neural mechanisms involved in mental imagery and observation of gait." Neuroimage 41(3): 1021-1031.

Jackson, P. L., M. F. Lafleur, et al. (2001). "Potential role of mental practice using motor imagery in neurologic rehabilitation." Arch Phys Med Rehabil 82(8): 1133-1141.

Jackson, P. L., M. F. Lafleur, et al. (2003). "Functional cerebral reorganization following motor sequence learning through mental practice with motor imagery." Neuroimage 20(2): 1171-1180.

Jeannerod, M. (1994). "The representing brain: neural correlates of motor intention and imagery." Behavioral and Brain Sciences 17: 187-245.

Jeannerod, M. and V. Frak (1999). "Mental imaging of motor activity in humans." Curr Opin Neurobiol 9(6): 735-739.

Johnson-Frey, S. H. (2004). "Stimulation through simulation? Motor imagery and functional reorganization in hemiplegic stroke patients." Brain Cogn 55(2): 328-331. 
Kaneko, F., T. Murakami, et al. (2003). "Decreased cortical excitability during motor imagery after disuse of an upper limb in humans." Clin Neurophysiol 114(12): 2397-2403.

Kimberley, T. J., G. Khandekar, et al. (2006). "Neural substrates for motor imagery in severe hemiparesis." Neurorehabil Neural Repair 20(2): 268-277.

Krakauer, J. W. and P. Mazzoni (2011). "Human sensorimotor learning: adaptation, skill, and beyond." Curr Opin Neurobiol 21(4): 636-644.

Kranczioch, C., S. Athanassiou, et al. (2008). "Shortterm learning of a visually guided power-grip task is associated with dynamic changes in EEG oscillatory activity." Clin Neurophysiol 119(6): 1419-1430.

Kranczioch, C., S. Mathews, et al. (2009). "On the equivalence of executed and imagined movements: evidence from lateralized motor and nonmotor potentials." Hum Brain Mapp 30(10): 3275-3286.

Kuhtz-Buschbeck, J. P., C. Mahnkopf, et al. (2003). "Effector-independent representations of simple and complex imagined finger movements: a combined fMRI and TMS study." Eur J Neurosci 18(12): 33753387.

Lafleur, M. F., P. L. Jackson, et al. (2002). "Motor learning produces parallel dynamic functional changes during the execution and imagination of sequential foot movements." Neuroimage 16(1): 142-157.

Lancaster, J. L., L. H. Rainey, et al. (1997). "Automated labeling of the human brain: a preliminary report on the development and evaluation of a forward-transform method." Hum Brain Mapp 5(4): 238-242.

Lancaster, J. L., M. G. Woldorff, et al. (2000) "Automated Talairach atlas labels for functional brain mapping." Hum Brain Mapp 10(3): 120-131.

Lehericy, S., E. Gerardin, et al. (2004). "Motor execution and imagination networks in post-stroke dystonia." Neuroreport 15(12): 1887-1890.

Lemon, R. N. (1999). "Neural control of dexterity: what has been achieved?" Exp Brain Res 128(1-2): 612.

Lemon, R. N. and R. Porter (1976). "Afferent input to movement-related precentral neurones in conscious monkeys." Proc R Soc Lond B Biol Sci 194(1116): 313-339.

Lewis, G. N. and W. D. Byblow (2004). "The effects of repetitive proprioceptive stimulation on corticomotor representation in intact and hemiplegic individuals." Clin Neurophysiol 115(4): 765-773.
Lindberg, P., C. Schmitz, et al. (2004). "Effects of passive-active movement training on upper limb motor function and cortical activation in chronic patients with stroke: a pilot study." J Rehabil Med 36(3): 117-123.

Lorey, B., S. Pilgramm, et al. (2011). "Activation of the parieto-premotor network is associated with vivid motor imagery--a parametric FMRI study." PLoS One 6(5): e20368.

Lotze, M., C. Braun, et al. (2003). "Motor learning elicited by voluntary drive." Brain 126(Pt 4): 866-872.

Lotze, M. and U. Halsband (2006). "Motor imagery." J Physiol Paris 99(4-6): 386-395.

Lotze, M., P. Montoya, et al. (1999). "Activation of cortical and cerebellar motor areas during executed and imagined hand movements: an fMRI study." J Cogn Neurosci 11(5): 491-501.

Lui, F., G. Buccino, et al. (2008). "Neural substrates for observing and imagining non-object-directed actions." Soc Neurosci 3(3-4): 261-275.

Macuga, K. L. and S. H. Frey (2012). "Neural representations involved in observed, imagined, and imitated actions are dissociable and hierarchically organized." Neuroimage 59(3): 2798-2807.

Mima, T., N. Sadato, et al. (1999). "Brain structures related to active and passive finger movements in man." Brain 122 ( Pt 10): 1989-1997.

Morin, O. and J. Grezes (2008). "What is "mirror" in the premotor cortex? A review." Neurophysiol Clin 38(3): 189-195.

Müller, K., C. M. Butefisch, et al. (2007). "Mental practice improves hand function after hemiparetic stroke." Restor Neurol Neurosci 25(5-6): 501-511.

Munzert, J., B. Lorey, et al. (2009). "Cognitive motor processes: the role of motor imagery in the study of motor representations." Brain Res Rev 60(2): 306-326.

Munzert, J., K. Zentgraf, et al. (2008). "Neural activation in cognitive motor processes: comparing motor imagery and observation of gymnastic movements." Exp Brain Res 188(3): 437-444.

Naito, E., H. H. Ehrsson, et al. (1999). "Illusory arm movements activate cortical motor areas: a positron emission tomography study." J Neurosci 19(14): 6134-6144.

Naito, E., P. E. Roland, et al. (2002). "I feel my hand moving: a new role of the primary motor cortex in somatic perception of limb movement." Neuron 36(5): 979-988. 
Nelles, G., G. Spiekramann, et al. (1999). "Evolution of functional reorganization in hemiplegic stroke: a serial positron emission tomographic activation study." Ann Neurol 46(6): 901-909.

Oldfield, R. C. (1971). "The assessment and analysis of handedness: The Edinburgh Inventory." Neuropsychologia 9: 97-113.

Page, S. J., P. Levine, et al. (2001). "A randomized efficacy and feasibility study of imagery in acute stroke." Clinical Rehabilitation 15: 233-240.

Page, S. J., J. P. Szaflarski, et al. (2009). "Cortical plasticity following motor skill learning during mental practice in stroke." Neurorehabil Neural Repair 23(4): 382-388.

Penhune, V. B. and C. J. Steele (2012). "Parallel contributions of cerebellar, striatal and M1 mechanisms to motor sequence learning." $\underline{\text { Behav }}$ Brain Res 226(2): 579-591.

Piefke, M., K. Kramer, et al. (2009). "Neurofunctional modulation of brain regions by distinct forms of motor cognition and movement features." Hum Brain Mapp 30(2): 432-451.

Pomeroy, V. M., C. A. Clark, et al. (2005). "The potential for utilizing the "mirror neurone system" to enhance recovery of the severely affected upper limb early after stroke: a review and hypothesis." Neurorehabil Neural Repair 19(1): 4-13.

Porro, C. A., M. P. Francescato, et al. (1996). "Primary motor and sensory cortex activation during motor performance and motor imagery: A functional magnetic resonance imaging study." Journal of Neuroscience 16(23): 7688-7698.

Puce, A., R. T. Constable, et al. (1995). "Functional magnetic resonance imaging of sensory and motor cortex: comparison with electrophysiological localization." J Neurosurg 83(2): 262-270.

Rizzolatti, G. and C. Sinigaglia (2010). "The functional role of the parieto-frontal mirror circuit: interpretations and misinterpretations." Nat Rev Neurosci 11(4): 264-274.

Roland, P. E. (1984). "Organization of motor control by the normal human brain." Hum Neurobiol 2(4): 205-216.

Roosink, M. and I. Zijdewind (2010). "Corticospinal excitability during observation and imagery of simple and complex hand tasks: implications for motor rehabilitation." Behav Brain Res 213(1): 35-41.

Sakreida, K., R. I. Schubotz, et al. (2005). "Motion class dependency in observers' motor areas revealed by functional magnetic resonance imaging." $\underline{\mathrm{J}}$ Neurosci 25(6): 1335-1342.

Sharma, N., J. C. Baron, et al. (2009). "Motor imagery after stroke: relating outcome to motor network connectivity." Ann Neurol 66(5): 604-616.

Sharma, N., P. S. Jones, et al. (2008). "Mapping the involvement of BA 4a and 4p during Motor Imagery." Neuroimage 41(1): 92-99.

Sharma, N., V. M. Pomeroy, et al. (2006). "Motor imagery: a backdoor to the motor system after stroke?" Stroke 37(7): 1941-1952.

Sharma, N., L. H. Simmons, et al. (2009). "Motor imagery after subcortical stroke: a functional magnetic resonance imaging study." Stroke 40(4): 1315-1324.

Shen, S., A. J. Szameitat, et al. (2008). "Detection of infarct lesions from single MRI modality using inconsistency between voxel intensity and spatial location--a 3-D automatic approach." IEEE Trans Inf Technol Biomed 12(4): 532-540.

Shima, K. and J. Tanji (2000). "Neuronal activity in the supplementary and presupplementary motor areas for temporal organization of multiple movements." Journal of Neurophysiology 84(4): 2148-2160.

Sirigu, A., J. R. Duhamel, et al. (1996). "The mental representation of hand movements after parietal cortex damage." Science 273(5281): 1564-1568.

Stefan, K., J. Classen, et al. (2008). "Concurrent action observation modulates practice-induced motor memory formation." Eur J Neurosci 27(3): 730-738.

Stephan, K. M., G. R. Fink, et al. (1995). "Functional anatomy of the mental representation of upper extremity movements in healthy subjects." Neurophysiol 73(1): 373-386.

Stinear, C. M., W. D. Byblow, et al. (2006). "Kinesthetic, but not visual, motor imagery modulates corticomotor excitability." Exp Brain Res 168(1-2): 157-164.

Stinear, C. M., M. K. Fleming, et al. (2007). "Lateralization of motor imagery following stroke." Clin Neurophysiol 118(8): 1794-1801.

Suminski, A. J., S. M. Rao, et al. (2007). "Neural and electromyographic correlates of wrist posture control." J Neurophysiol 97(2): 1527-1545.

Szameitat, A. J., S. Shen, et al. (2007). "Effectordependent activity in the left dorsal premotor cortex in motor imagery." Eur J Neurosci 26(11): 3303-3308. 
Szameitat, A. J., S. Shen, et al. (2007). "Motor imagery of complex everyday movements. An fMRI study." Neuroimage 34(2): 702-713.

Talairach, P. and J. Tournoux (1988). A Stereotactic Coplanar Atlas of the Human Brain. Stuttgart, Thieme.

Terumitsu, M., K. Ikeda, et al. (2009). "Participation of primary motor cortex area $4 \mathrm{a}$ in complex sensory processing: 3.0-T fMRI study." Neuroreport 20(7): 679-683.

Thickbroom, G. W., B. A. Phillips, et al. (1998). "Isometric force-related activity in sensorimotor cortex measured with functional MRI." Exp Brain Res 121(1): 59-64.

Tombari, D., I. Loubinoux, et al. (2004). "A longitudinal fMRI study: in recovering and then in clinically stable sub-cortical stroke patients." Neuroimage 23(3): 827-839.

Wang, C., Y. Wai, et al. (2008). "The cortical modulation from the external cues during gait observation and imagination." Neurosci Lett 443(3): 232-235

Ward, N. S. (2004). "Functional reorganization of the cerebral motor system after stroke." Curr Opin Neurol 17(6): 725-730.

Ward, N. S., M. M. Brown, et al. (2003). "Neural correlates of outcome after stroke: a cross-sectional fMRI study." Brain 126(Pt 6): 1430-1448.

Ward, N. S., M. M. Brown, et al. (2006). "Longitudinal changes in cerebral response to proprioceptive input in individual patients after stroke: an FMRI study." Neurorehabil Neural Repair 20(3): 398-405.

Weiller, C., M. Juptner, et al. (1996). "Brain representation of active and passive movements." Neuroimage 4(2): 105-110.

Wolpert, D. M. and M. Kawato (1998). "Multiple paired forward and inverse models for motor control." Neural Netw 11(7-8): 1317-1329.

Yetkin, F. Z., W. M. Mueller, et al. (1995). "Functional magnetic resonance imaging mapping of the sensorimotor cortex with tactile stimulation." Neurosurgery 36(5): 921-925.

\section{Acknowledgements}

This study was funded by the Medical Research Council, UK (CEG 61501; Dr Sterr). We thank Ari Lingeswaran for assistance in data acquisition. 
Table 1. Patient demographics and lesion details.

\begin{tabular}{|c|c|c|c|c|c|c|c|c|c|}
\hline 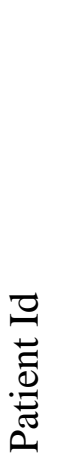 & 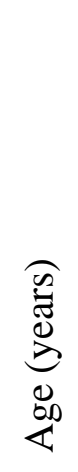 & 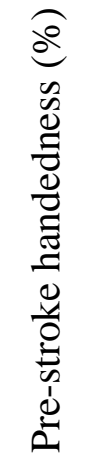 & $\begin{array}{l}\frac{\dot{\theta}}{\mathbb{8}} \\
\stackrel{0}{0}\end{array}$ & 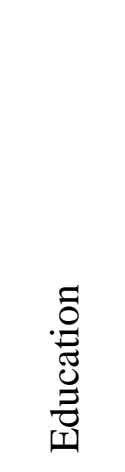 & 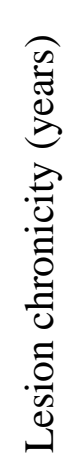 & 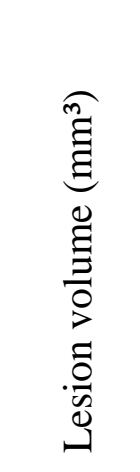 & 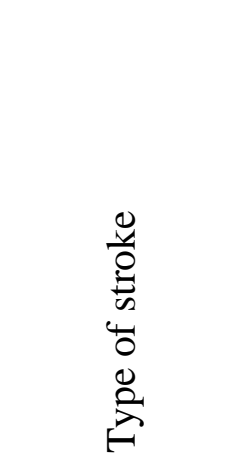 & 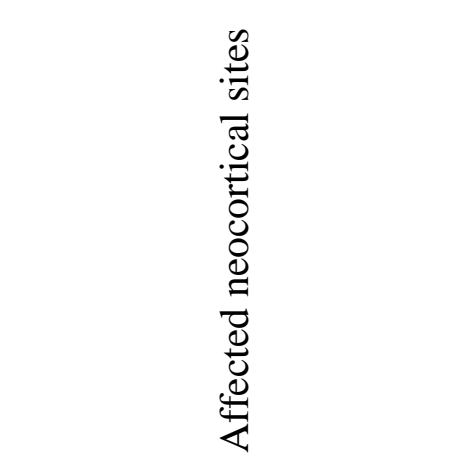 & 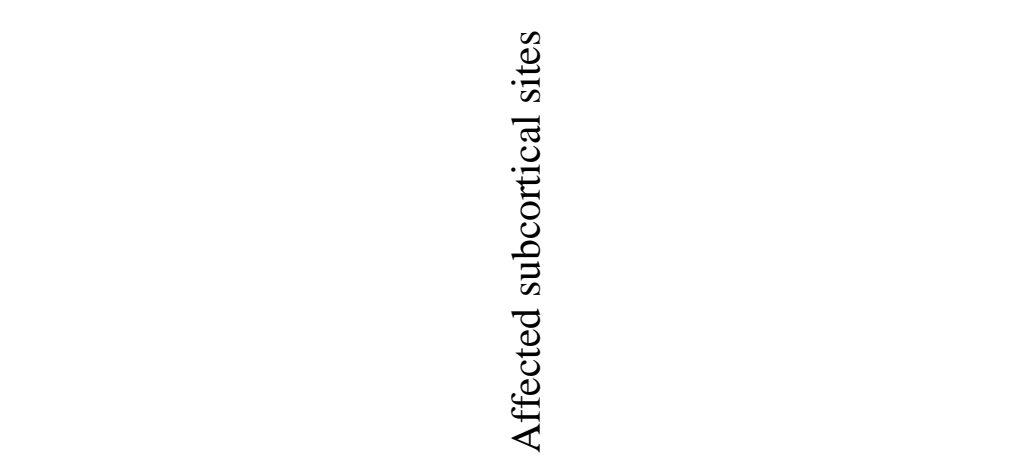 \\
\hline 1 & 64 & 100 & $\mathrm{~m}$ & $\mathrm{PhD}$ & 4 & 42277 & ischemic & frontal, temporal, parietal & basal ganglia, insula, corticospinal tract \\
\hline 2 & 61 & 100 & $\mathrm{f}$ & $10 \mathrm{yr}$ & 3 & 35060 & ischemic & frontal, temporal & basal ganglia, insula \\
\hline 3 & 61 & 100 & $\mathrm{~m}$ & $10 \mathrm{yr}$ & 6 & 71018 & ischemic & $\begin{array}{l}\text { frontal, temporal, } \\
\text { parietal, occipital }\end{array}$ & basal ganglia, thalamus, insula \\
\hline 4 & 57 & 100 & $\mathrm{~m}$ & B.Sc. & 11 & 1506 & hemorrhagic & none & $\begin{array}{c}\text { putamen, globus pallidus, posterior limb of external capsule, } \\
\text { centrum semiovale, insula, thalamus }\end{array}$ \\
\hline 5 & 67 & 90 & $\mathrm{f}$ & $\begin{array}{c}\mathrm{O}- \\
\text { level }\end{array}$ & 1 & 1589 & hemorragic & none & putamen, globus pallidus, centrum semiovale \\
\hline
\end{tabular}


Table 2. Patient behavioral results.

\begin{tabular}{|c|c|c|c|c|c|c|c|c|c|c|c|c|c|c|}
\hline 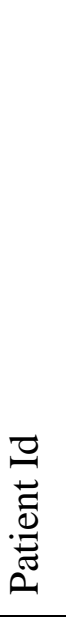 & 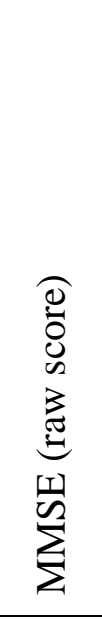 & 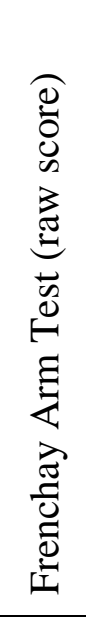 & 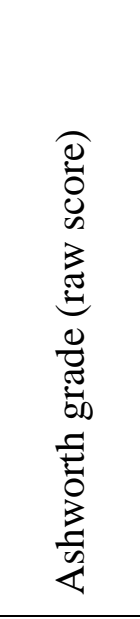 & 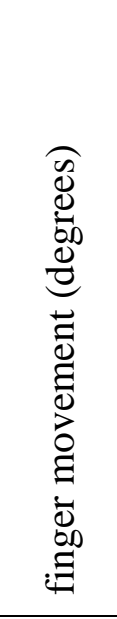 & 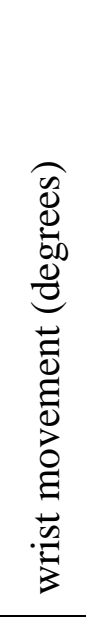 & 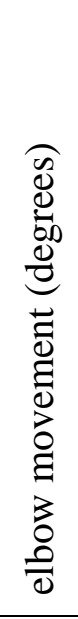 & 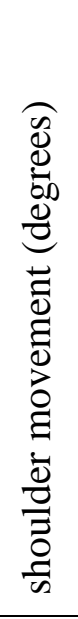 & 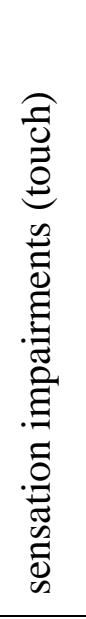 & 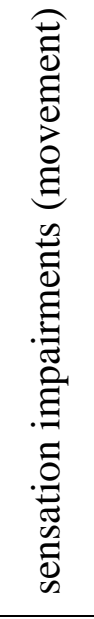 & 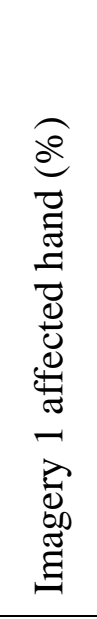 & 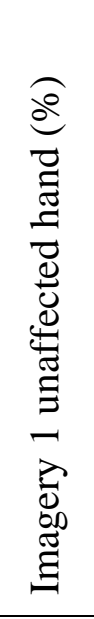 & 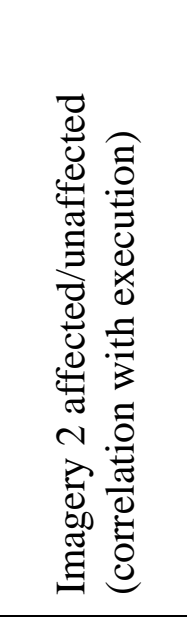 & 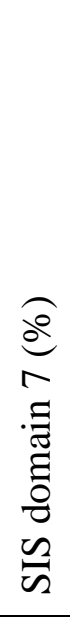 & 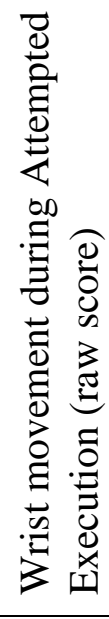 \\
\hline 1 & 30 & $2^{(1)}$ & 4 & 0 & $<5$ & 0 & 50 & $\mathrm{fl}$ & fwe & 100 & 100 & $.14 / .94^{* *}$ & 0 & 1 \\
\hline 2 & 30 & $2^{(1)}$ & $2-3$ & $1-10$ & 0 & 20 & 20 & - & - & 67 & 100 & $.93^{* *} /-.04$ & 0 & 0.33 \\
\hline 3 & 26 & $2^{(1)}$ & $4-5$ & $<5$ & 5 & 75 & 70 & fw & fw & 83 & 83 & $.79 / .97^{* *}$ & 0 & 0.83 \\
\hline 4 & 30 & 0 & 4 & 0 & 0 & 10 & $<5$ & $f$ & 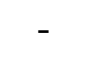 & 100 & 100 & $.59 / .76$ & 0 & 0.5 \\
\hline 5 & 30 & $2^{(1)}$ & $3-3.5$ & 0 & 5 & 0 & $<5$ & - & - & 100 & 100 & $-.27 / .78$ & 5 & 0.17 \\
\hline$e d$ & 29.2 & 1.6 & 3.4-3.9 & $1.2-3$ & 3 & 21 & 30 & & & 90 & 97 & $.64 / .96^{* *}$ & 1 & .57 \\
\hline
\end{tabular}

Notes

${ }^{(1)}$ Patient was able to draw an almost straight line with the healthy hand using the affected hand to fixate the ruler on the table. Sensation impairments: - no impairments; impairments in f finger, $w$ wrist, 1 lower arm, e elbow.

Imagery 2 refers to the correlation coefficients of overt execution of the movements (unaffected hand) with affected hand imagery (first value) and unaffected hand imagery (second value). Low correlation indicates impairments with kinesthetic motor imagery. Mean correlation based on mean of raw data. ** significant with $\mathrm{p}<.01$

SIS domain 7: Abilities to use affected hand

Wrist movement refers to average movement of all 6 attempted execution blocks on a scale 0 [no movement] to 5 [strong movement]. 
Table 3. Anatomical location, cytoarchitectonic areas (in brackets), MNI coordinates, and t values of sensorimotor activation peaks for the baseline comparisons in HVs.

Cytoarchitectonic areas were determined by the Anatomy toolbox (no superscript), by the Talairach demon $\left({ }^{1}\right)$ or manually by visual inspection $\left({ }^{2}\right)$. All $p<.05$ (FWE corrected).

Location

$\mathbf{x}, \mathbf{y}, \mathbf{z} \quad \mathbf{T}$

Execution

\begin{tabular}{lcc}
\hline L preCG (4) & $-38,-28,70$ & 15,19 \\
L rol op (OP1) & $-46,-24,16$ & 10,29 \\
R rol op (44) & $48,4,10$ & 9,74 \\
R rol op (OP1) & $56,-26,20$ & 8,87 \\
L postCG (4p, 3b, 4a) & $-36,-24,54$ & 13,15 \\
L postCG $(1,2)$ & $-46,-28,54$ & 12,3 \\
L postCG $(1,2)$ & $-26,-40,68$ & 9,53 \\
L SMA (6) & $-4,-10,64$ & 12,11 \\
L SMA (6, 4a) & $-4,-20,48$ & 9
\end{tabular}

Passive Movement

\begin{tabular}{lcc}
\hline R preCG (44) & $50,4,10$ & 6.62 \\
L rol op (OP1) & $-48,-26,18$ & 9.40 \\
R rol op (OP1) & $56,-24,18$ & 9.30 \\
L preCG (4a, 1) & $-36,-30,62$ & 13.75 \\
L preCG (6) & $-32,-14,58$ & 7.57 \\
L preCG (6) & $-26,-16,66$ & 7.52 \\
L postCG (3b, 2, 1) & $-26,-40,60$ & 10.95 \\
L SMA (6) & $-4,-10,62$ & 11.97 \\
R SMA (6) & $8,-10,66$ & 6.92 \\
R SMA (6) & $12,6,46$ & 6.39 \\
L parac lob (4a, 6) & $-4,-24,46$ & 11.31 \\
L IPL (2, 1) & $-56,-22,46$ & 8.82
\end{tabular}

Imagery

\begin{tabular}{|c|c|c|}
\hline L IFG $(44)^{2}$ & $-52,8,2$ & 7,12 \\
\hline R IFG (44) & $50,12,8$ & 6,98 \\
\hline L SFG $(6)^{2}$ & $-18,-6,54$ & 7,13 \\
\hline L preCG (44) & $-52,8,14$ & 6,56 \\
\hline L preCG (6) & $-54,6,38$ & 8,02 \\
\hline L SMA (6) & $-8,-10,64$ & 9,28 \\
\hline R SMA $(6)^{2}$ & $12,4,48$ & 7,67 \\
\hline R SMA (6) & $6,-10,66$ & 6,59 \\
\hline
\end{tabular}

Observation

\begin{tabular}{lll}
\hline R MFG $(6)^{1}$ & $38,-4,68$ & 7,13 \\
R preCG (6) & $48,-2,58$ & 6,83 \\
L preCG (6) & $-54,6,38$ & 7,11 \\
L IPL (2) & $-46,-40,50$ & 6,96
\end{tabular}


Table 4. Anatomical location, cytoarchitectonic areas (in brackets), MNI coordinates, and t values of sensorimotor activation peaks for the baseline comparisons in patients. Cons refers to consistency of activations across patients (for instance, ,,5-4 (3-2)“ shows that 5 patients showed an effect ( 4 of them significant) in a $5 \times 5 \times 5$ voxel cube around the group peak, 3 patients showed an effect ( 2 of them significant) directly at the peak voxel of the group peak; see also Methods section for details). For further details see Table 3.

\begin{tabular}{llll} 
Location & $\mathbf{x}, \mathbf{y}, \mathbf{z}$ & $\mathbf{T}$ & Cons \\
\hline & & & \\
Execution & & & \\
\hline L IFG (44) & $-44,14,4$ & 8.30 & $5-4(5-4)$ \\
R SFG (6) & $18,-10,68$ & 5.27 & $5-5(4-2)$ \\
R preCG (6) & $32,-18,62$ & 5.01 & $5-4(3-3)$ \\
R preCG (6) & $24,-30,70$ & 9.80 & $5-4(5-3)$ \\
L preCG (6) & $-40,-10,48$ & 5.54 & $5-4(5-4)$ \\
L preCG (4a, 6) & $-36,-18,56$ & 6.58 & $5-4(4-4)$ \\
L postCG (4a) & $-48,-14,48$ & 6.85 & $5-4(4-4)$ \\
L postCG (2) & $-32,-36,50$ & 4.81 & $4-4(4-4)$ \\
L postCG (4p) & $-20,-38,54$ & 5.27 & $4-4(4-3)$ \\
R postCG (1, 4a) & $34,-32,64$ & 10.90 & $5-5(4-3)$ \\
R postCG (2) & $30,-36,46$ & 5.50 & $4-4(4-2)$ \\
L parac lob (6) & $-6,-16,72$ & 5.57 & $5-5(5-2)$ \\
L parac lob (6) & $0,-22,58$ & 9.36 & $4-4(4-4)$ \\
L parac lob (6, 4a) & $-8,-28,74$ & 7.36 & $5-5(5-3)$ \\
R parac lob (6) & $4,-24,72$ & 5.39 & $5-5(5-5)$ \\
R parac lob (4a) & $0,-30,68$ & 6.46 & $5-4(5-4)$ \\
R parac lob (4a) & $6,-42,68$ & 6.49 & $5-5(4-3)$ \\
L rol op (44) & $-52,2,14$ & 4.83 & $5-4(5-2)$ \\
L rol op (OP4) & $-58,-2,4$ & 7.44 & $5-5(5-4)$ \\
L rol op (OP4) & $-48,-8,8$ & 5.84 & $5-4(4-3)$ \\
\end{tabular}

Passive Movement

R med SFG (6) 8, -24, $48 \quad 7.01 \quad 5-4(4-3)$

$\mathrm{R}$ postCG $(1,2,3 \mathrm{~b}, 4 \mathrm{a}, 4 \mathrm{p}) \quad 38,-34,60 \quad 18.58 \quad 5-5(5-5)$

Imagery

\begin{tabular}{|c|c|c|c|}
\hline L IFG $(44)^{1}$ & $-60,4,10$ & 5.72 & 4-4 (4-4) \\
\hline L IFG $(44)^{2}$ & $-56,10,0$ & 6.67 & $4-4(4-3)$ \\
\hline R preCG $(6,4 a)$ & $54,-8,40$ & 5.30 & $5-5(5-3)$ \\
\hline R preCG (6) & $36,-16,62$ & 5.72 & $5-4(5-2)$ \\
\hline R preCG (6) & $12,-24,74$ & 5.55 & $5-3(3-2)$ \\
\hline R preCG $(6,4 a)$ & $22,-30,72$ & 8.10 & $5-3(3-2)$ \\
\hline L preCG $(6,44)^{2}$ & $-52,2,32$ & 6.20 & $5-3(4-2)$ \\
\hline L preCG (6) & $-24,-22,68$ & 4.80 & $5-3(3-2)$ \\
\hline L preCG (6) & $-60,0,20$ & 6.28 & $4-4(4-3)$ \\
\hline L postCG (4a) & $-52,-12,44$ & 6.31 & $4-4(4-3)$ \\
\hline R postCG (3b) & $60,-2,20$ & 5.48 & $5-4(5-3)$ \\
\hline R postCG (3b) & $44,-26,56$ & 7.33 & $5-2(5-2)$ \\
\hline $\mathrm{R}$ postCG (4a) & $32,-32,64$ & 9.77 & $5-4(4-2)$ \\
\hline R SPL (2) & $34,-50,62$ & 5.12 & $5-3(3-2)$ \\
\hline $\operatorname{LSMA}(6,4 a)$ & $0,-18,54$ & 9.97 & $5-3(4-3)$ \\
\hline L parac lob $(6,4 a)$ & $-8,-28,74$ & 7.01 & $4-3(4-2)$ \\
\hline \multicolumn{4}{|l|}{ Observation } \\
\hline R preCG $(6)^{1}$ & $32,-8,52$ & 4.88 & $5-4(5-3)$ \\
\hline
\end{tabular}




$\begin{array}{llll}\text { R preCG (6) } & 46,-8,52 & 5.02 & 5-3(4-2) \\ \text { R preCG (6) } & 38,-16,60 & 5.64 & 5-4(5-3) \\ \text { L SMA (6) } & -6,-8,54 & 5.00 & 5-4(5-2) \\ \text { L SMA (6) } & -8,-12,64 & 5.00 & 5-4(3-3) \\ \text { R SMA (6) } & 2,-8,56 & 5.08 & 5-3(5-2) \\ \text { L parac lob (6) } & -6,-16,72 & 4.75 & 5-5(5-1) \\ \text { R IPL (2) } & 32,-44,52 & 4.82 & 5-5(5-3)\end{array}$

\section{Figures}

Figure 1. Schematic illustration of the manipulandum. Participants' hands were fixed on the flap using Velcro straps (not shown). At rest, the flap rested on the force sensors.

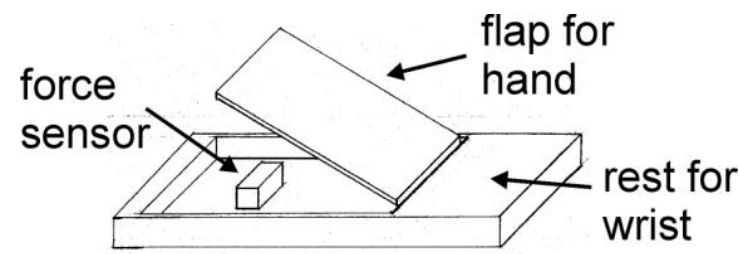

Figure 2. Illustration of movie sequence (1 cycle, lasting $1 \mathrm{~s}$ ) used in the Observation condition. This cyclic wrist flexion-extension movement was the basis of all conditions. Each block consisted of 20 cycles.

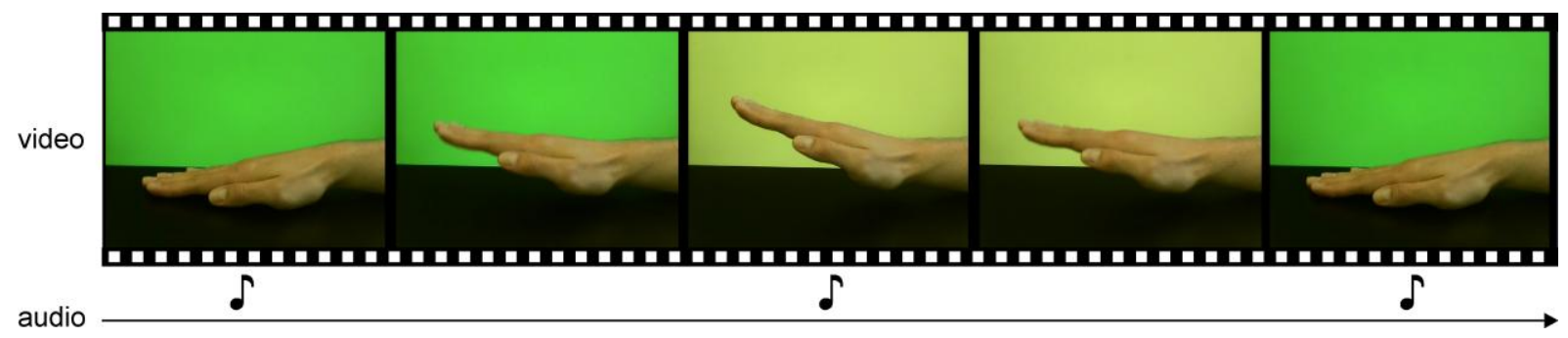


Figure 3. Patients' anatomical MRI scans illustrating lesion site and volume.

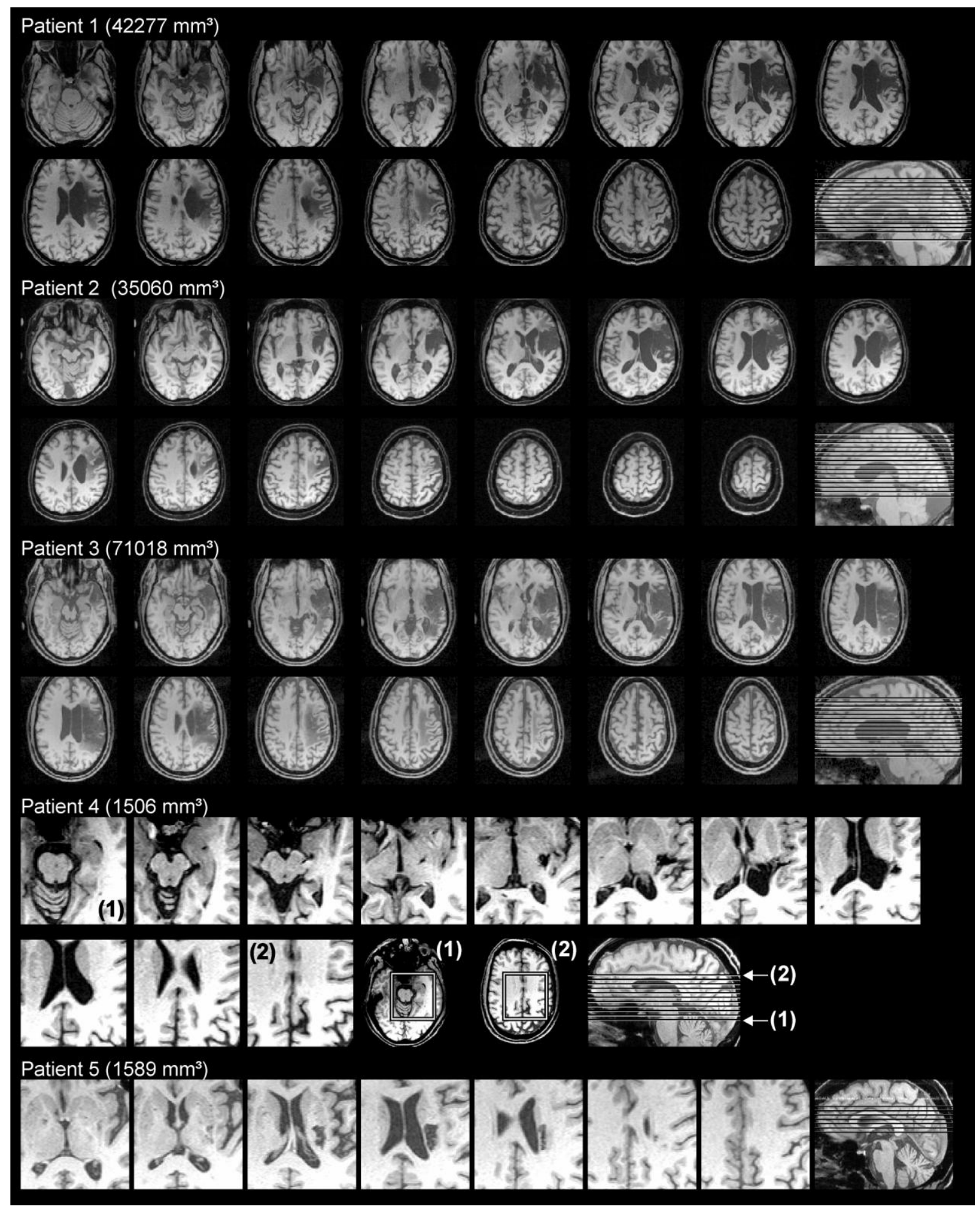


Figure 4. MRI results of baseline comparisons. (A) Healthy volunteers $(\mathrm{N}=21)$ performed the task with the right hand. Data were analyzed using a random effects model. (B) Patients $(\mathrm{N}=5)$ performed the task with the left hand, i.e. their affected hand. Data were analyzed using a fixed-effect model. All maps thresholded at $\mathrm{p}<0.05$, FWE-corrected for multiple comparisons. Participants had their eyes closed during all conditions except for Observation and Baseline $(\mathrm{O})$.

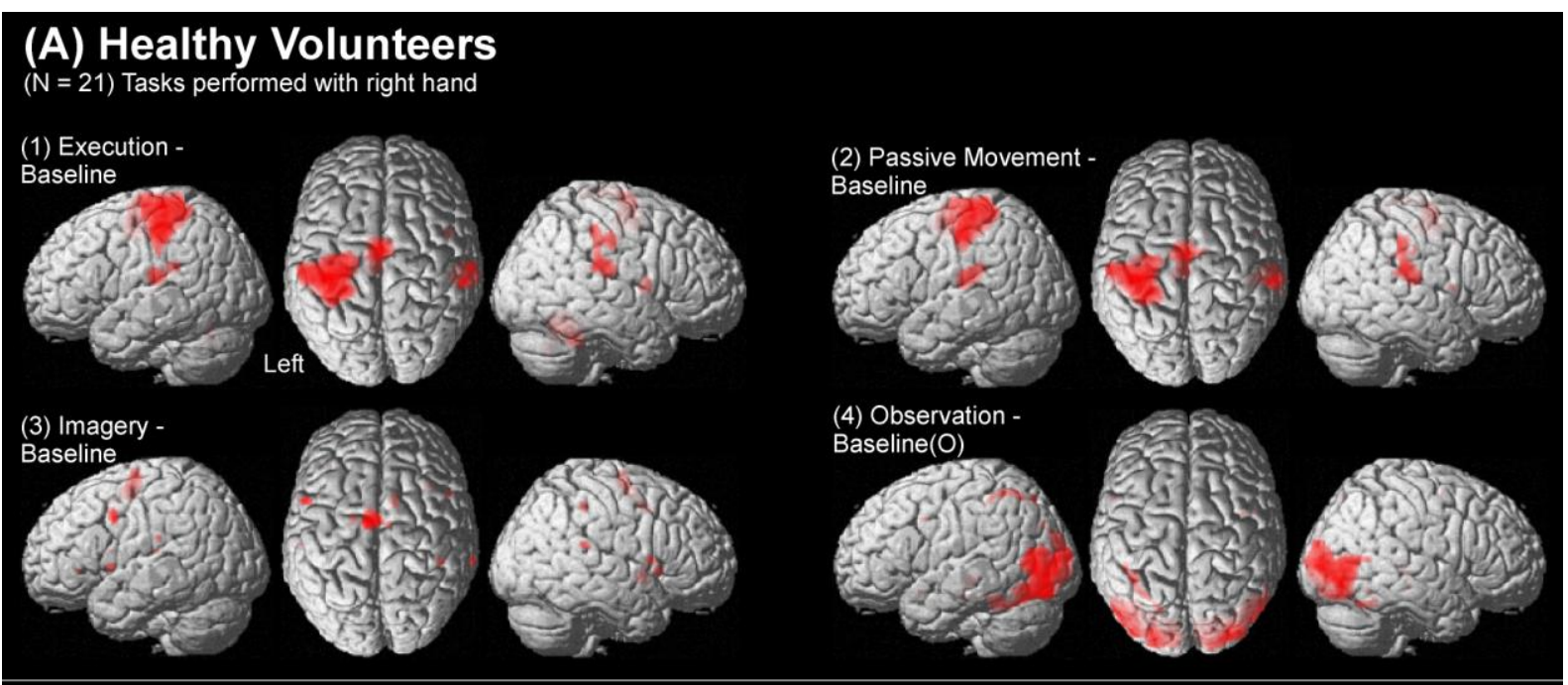

\section{(B) Patients}

$(\mathrm{N}=5)$ Tasks performed with left hand (affected hand)
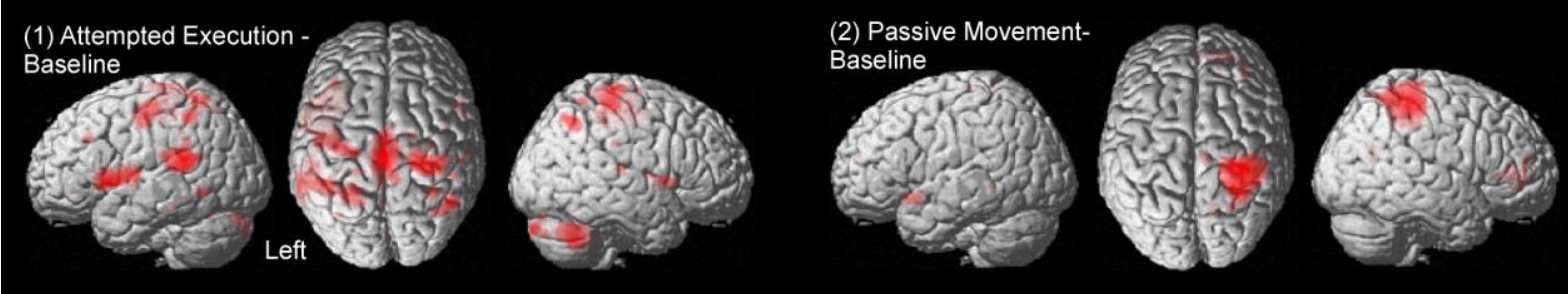

(3) Imagery -

Baseline
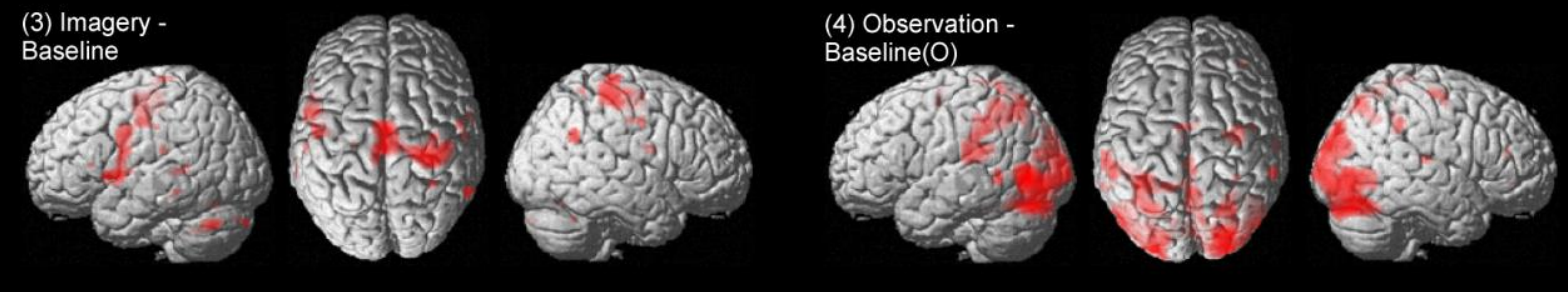